\title{
Targeting the TP53/MDM2 axis enhances radiation sensitivity in atypical teratoid rhabdoid tumors
}

\author{
IRINA ALIMOVA $^{1}$, DONG WANG ${ }^{1}$, ETIENNE DANIS $^{1}$, ANGELA PIERCE $^{1}$, ANDREW DONSON $^{1,2}$, \\ NATALIE SERKOVA $^{3}$, KRISHNA MADHAVAN ${ }^{1}$, SENTHILNATH LAKSHMANACHETTY ${ }^{1}$, \\ ILANGO BALAKRISHNAN ${ }^{1}$, NICHOLAS K. FOREMAN ${ }^{1,2,4,5}$, SIDDHARTHA MITRA ${ }^{1,2}$, \\ SUJATHA VENKATARAMAN ${ }^{1,2}$ and RAJEEV VIBHAKAR ${ }^{1,2,4,5}$ \\ ${ }^{1}$ Department of Pediatrics, University of Colorado Anschutz Medical Campus; \\ ${ }^{2}$ Morgan Adams Foundation Pediatric Brain Tumor Research Program, Children's Hospital Colorado; \\ ${ }^{3}$ Department of Radiology, University of Colorado School of Medicine; ${ }^{4}$ Center for Cancer and Blood Disorders, \\ Children's Hospital Colorado; ${ }^{5}$ Department of Neurosurgery, University of Colorado Denver, Aurora, CO 80045, USA
}

Received November 8, 2021; Accepted January 17, 2022

DOI: $10.3892 / \mathrm{ijo} .2022 .5322$

\begin{abstract}
Atypical teratoid rhabdoid tumor (ATRT) is a highly aggressive pediatric brain tumor. Despite radiation, aggressive chemotherapy and autologous stem cell rescue, children usually have a poor survival time. In the present study, the role of TP53/MDM2 interaction in ATRT was investigated. A functional genomic screen identified the TP53/MDM2 axis as a therapeutic target in the central nervous system (CNS) ATRT. Gene expression analysis revealed that all ATRT sub-groups expressed high levels of MDM2, which is a negative regulator of TP53. Using cell viability, colony formation and methylcellulose assays it was found that genetic MDM2 inhibition with short hairpin RNA or chemical MDM2 inhibition with small molecule inhibitors, Nutlin3 and idasanutlin (RG7388) decreased the growth of ATRT cell lines. Furthermore, idasanutlin significantly decreased the growth of intracranial orthotopic ATRT brain tumors, as evaluated using T2 MRI, and prolonged survival time relative to control animals. MRI of intracranial tumors showed that diffusion coefficient, an effective marker for successful treatment, significantly increased with idasanutlin treatment showing tumor necrosis/apoptosis. Immunohistochemistry revealed an increased number of caspase-3-positive cells in the idasanutlin treatment group, confirming the induction of apoptosis in vivo. Using flow
\end{abstract}

Correspondence to: Dr Rajeev Vibhakar, Department of Pediatrics, University of Colorado Anschutz Medical Campus, 12800 East 19th Ave, RC1-North Building, Aurora, CO 80045, USA E-mail: rajeev.vibhakar@cuanschutz.edu

Abbreviations: ATRT, atypical teratoid rhabdoid tumor; ADC, apparent diffusion coefficient; CFA, colony formation assay

Key words: ATRT, SMARCB1, MDM2, TP53, idasanutlin, radiation sensitivity cytometry and western blot analysis we show that inhibition of MDM2 enhanced radiation sensitivity in vitro by potentiating DNA damage via the induction of the TP53/Bax/Puma proapoptotic axis. Furthermore, DNA damage was associated with increased mitochondrial reactive oxygen species accumulation. The present study demonstrated that MDM2 expression level was increased in ATRT patient samples and MDM2 inhibition suppressed ATRT cell growth in vitro, and leads to apoptosis in vivo. MDM2 inhibition potentiates DNA damage and sensitizes ATRT cells to radiation. These findings highlight the TP53/MDM2 axis as a rational therapeutic target in CNS ATRT.

\section{Introduction}

Atypical teratoid rhabdoid tumor (ATRT) is a fast growing, highly malignant brain tumor in childhood (infants to $<18$ years old), with a poor survival rate worldwide, between 1996 and 2020 (5-year survival, 35-40\%) (1,2). Current therapy, including intensive chemotherapy, radiation and autologous stem cell transplant, are suboptimal and therapy-related toxicity remains a major concern in this young age group $(3,4)$. Currently, there is no standard treatment for ATRTs, with few ATRT prospective studies performed and a lack of novel targeted therapies (2-4). The most widely accepted therapeutic option is the use of high-dose chemotherapy, with stem cell rescue, followed by radiotherapy in infants and young children ( $<5$ years old) and the use of intensive multimodal chemotherapy with radiation in patients who are older (6-18 years old) (2).

ATRT's main molecular feature is the loss of the SMARCB1 gene, resulting in the epigenomic dysregulation of the genome (5,6). SMARCB1, a member of the switch/sucrose non-fermentable (SWI/SNF) chromatin remodeling complex, affects lineage commitment and differentiation by controlling gene expression (7). ATRTs demonstrate a quiescent structural genome, but are heterogeneous in gene expression (8-11). The paucity of structural genetic events creates a significant barrier 
to the development of novel targeted therapy. Nevertheless, the transcriptional heterogeneity represents an opportunity to examine novel approaches to therapy. Current international consensus groups ATRT into 3 transcriptomic groups: ATRT-TYR, ATRT-SHH and ATRT-MYC $(12,13)$. Associated SMARCB1 deficient tumors, occurring outside of the central nervous system are known as malignant rhabdoid tumors (MRT) and exhibit distinct gene expression signatures, further adding to the heterogeneity (14). However, these molecular data are not yet used to guide therapy.

ATRT, present with wild-type (WT) TP53, indicated that targeting negative regulators of TP53 could represent a novel therapeutic strategy. Reconstitution of a functional TP53 pathway is an attractive antitumor strategy. TP53 is negatively regulated by MDM2, an ubiquitin ligase, that targets TP53 for proteolytic degradation (15). The associated MDM4 sequesters TP53 and inhibits its transcription transactivation activity (15). Over the past 2 decades numerous effective inhibitors of MDM2 have been developed (16,17). MDM2/TP53 binding antagonists, a novel class of anti-cancer therapeutics currently in early clinical development, non-genotoxically activate WT TP53 by disrupting the interaction between TP53 and MDM2 (17). The first member of the nutlins family, Nutlin3, demonstrated strong activity in animal models with limited toxicity (18).

Idasanutlin (RG7388), a second generation MDM2/TP53 inhibitor, is available in both oral and intravenous formulations, and shows increased bioavailability and selectivity (19). Idasanutlin demonstrates antitumor activity in pediatric cancers, including neuroblastoma and sarcoma $(20,21)$. Previous studies demonstrated that TP53 was a negative regulator of SMARCB1deficient cancers cell growth and that targeting MDM2 could reactivate the TP53 pathway in MRT $(22,23)$.

In the present study it was found that inhibition of MDM2 potentiates TP53 to suppress intra-cranial ATRT in vivo, and enhances sensitivity to radiation in vitro by enhancing DNA damage.

\section{Materials and methods}

Study approval. Primary patient sample (from brain surgery; 12-month-old male) was obtained from the Children's Hospital Colorado (Colorado, USA) in February, 2010. The sample was collected in accordance with local and Federal human research protection guidelines and institutional review board regulations (approval no, COMIRB 95-500). Informed consent was provided by all patients or from a parent or guardian. The MAF737 (TYR) SMARCB1 INI1-deleted cell line was established from the sample.

Cell lines and reagents. All the cell lines were cultered in a cell incubator (Thermo Fisher Scientific, Inc.) at $37^{\circ} \mathrm{C}$ with 5\% $\mathrm{CO}_{2}$. The BT16 ATRT (TYR 2A/MYC) cell line was a gift from Dr Peter Houghton (Nationwide Children's Hospital, Center for Childhood Cancer and Blood Diseases, OH, USA). The MAF737 (TYR), SMARCB1 INI1-deleted cell line was established from a surgical sample of a 12-month-old male. The sample was obtained from the Children's Hospital Colorado (Colorado, USA) and collected in accordance with local and Federal human research protection guidelines and IRB regulations (approval no. COMIRB 95-500) (11). Consent was provided by a parent. The cells were cultured in RPMI medium (Gibco; Thermo Fisher Scientific, Inc.) supplemented with 10\% FBS (Atlanta Biologicals, Inc.) and 1\% Pen/Strep (Gibco; Thermo Fisher Scientific, Inc.) $(24,25)$.

The CHB-ATRT1 cell line were derived from a tumor obtained at the time of primary surgical resection of a posterior fossa ATRT at Boston Children's Hospital and kindly provided by Dr Mitra (University of Colorado Anschutz Medical Campus, CO, USA) and Dr Yoon Jae-Cho (Stanford University School of Medicine, CA, USA). The CHB-ATRT1 cell line has de-repression of GLI1 transcription via the loss of SNF5/SMARCB1/INI1(26). The CHB-ATRT1 cell line was cultured in Neurobasal medium (Gibco; Thermo Fisher Scientific, Inc.), with epidermal growth factor and leukemia inhibitory factor (26).

The CHLA04 (ATRT/SHH) cell line was purchased from American Type Culture Collection and was cultered in DMEM/F12 medium (Gibco; Thermo Fisher Scientific, Inc.) with epidermal growth factor (VWR International, LLC) and 2\% B27 supplement (Gibco; Thermo Fisher Scientific, Inc.) Immortalyzed normal human astrocytes (NHA) were provided by Dr Cynthia Hawkins (Sick Kids Hospital, Department of Pediatric Laboratory Medicine, Toronto, Canada). The NHA cell line was cultered in DMEM (Gibco; Thermo Fisher Scientific, Inc.) supplemented with $10 \%$ FBS (Atlanta Biologicals, Inc.), and $1 \%$ Pen/Strep, L-glutamine and sodium pyruvate (Gibco; Thermo Fisher Scientific, Inc.) All the cells were cultured according to provided protocols.

All the cell lines were routinely tested for mycoplasma contamination. Idasanutlin (RG7388; cat. no. HY-15676) and Nutlin3 (cat. no. HY-50696) were purchased from Med Chem Express and reconstituted in dimethyl sulfoxide (DMSO; cat. no. D2650; Sigma-Aldrich; Merck KGaA). DMSO was used as a control.

Functional genomic short hairpin (sh)RNA screening. Functional genomic shRNA screening was performed as previously described (27). The MAF737 cell line was transduced for $24 \mathrm{~h}$ with a pooled lentivirus shRNA library, consisting of 4,200 shRNAs targeting 408 epigenetic genes (backbone, Human PLVX-ZsGreen lentiviral shRNA-mir; cat. no. TRH6110; TransOMIC Technologies, Inc.). Plasmid DNA $(2.5 \mu \mathrm{g})$ and Lipofectamine ${ }^{\circledR} 3000(7.5 \mu \mathrm{l}$; cat. no. 100022050; Invitrogen; Thermo Fisher Scientific, Inc.) was used for transfection at $37^{\circ} \mathrm{C}$.

Genomic DNA was isolated (DNeasy blood and tissue kit; cat. no. 69504; Qiagen $\mathrm{GmbH}$ ) from the transduced cells 4 and 21 days after transduction. The quality of the samples was verified using a TapeStation 4200 (Agilent Technologies, Inc.). Then, the samples were processed to amplify individual shRNAs with two rounds of PCR, and sequenced with an Illumina Hiseq instrument, with single read for 50 cycles. 3 base pair tag length, 5' to $3^{\prime}$. The loading concentration of the final library was $10 \mathrm{nM}$. The concentration was measured using a Fluorometer Qubit 2.0 (Invitrogen; Thermo Fisher Scientific, Inc.). The sequencing kit was purchased from transOMIC Technologies, Inc. (cat. no. TRP 0001). 
The sequencing results were analyzed using R-based package DESEq2 (v1.34.0; Bioconductor.org). By comparing the shRNAs present on day 4 to 21 , with a false discovery rate of 0.5 and 0.1 , respectively (27).

Gene expression analysis. The gene expression data of 42 ATRT tumor samples and 9 normal cerebellum samples were analyzed from the public dataset, GSE70678 (9) using R (v4.0.5; https://www.r-project.org/). The affy package (v1.44.0) (28) and the custom chip definition file (hugene11sthsensgcdf; v25.0.0; http://brainarray.mbni.med.umich. edu/brainarray/database/customCDF/25.0.0/ensg.asp) (29) was used to load, and summarize the expression level of genes and process the data. The samples flagged by the arrayQualityMetrics Bioconductor package (v3.22.0) (30) were removed due to low quality. Expression data were normalized using the Robust Multichip Average method (31). Kruskal-Wallis test was used to analyze the data between normal samples and each subtype. $\mathrm{P}<0.05$ was considered to indicate a statistically significant difference.

Transfection of ATRT cells. The ATRT cell lines were transfected with plasmids: shTP53 (cloneID, TRCN00003755; pLKO.1; 5'-CCGGGTCCAGATGAAGCTCCCAGAACTCGA GTTCTGGGAGCTTCATCTGGACTTTTTG-3'), shMDM2 (Clone ID, TRCN0000355725; pLKO.1; 5'-CCGGATTATCTG GTGAACGACAAAGCTCGAGCTTTGTCGTTCACCAGA TAATTTTTTG-3'), TP53 human open reading frame (clone ID, ccsbBroad304_07088; Backbone, pLX304), shC202 (puro-non-mamallian; pLKO.5; 5'-CCGGCAACAAGATGA AGAGCACCAACTCGAGTTGGTGCTCTTCATCTTGT-3') (all from Functional Genomics Shared Resource, University of Colorado). A total of $2.5 \mu \mathrm{g}$ plasmid DNA and $7.5 \mu \mathrm{l}$ Lipofectamine ${ }^{\circledR} 3000$ (cat. no. 100022050 ; Invitrogen; Thermo Fisher Scientific, Inc.) was used for transfection at $37^{\circ} \mathrm{C}$. The cells were selected with puromicin $(1 \mu \mathrm{g} / \mathrm{ml})$ for $48 \mathrm{~h}, 24 \mathrm{~h}$ following transfection. The experiments were performed the following day after puromicin selection. Empty plasmid was used as the control in all cases.

Luciferase-expressing MAF737 ATRT cell line was obtained by transfecting with the pLV[Exp]-Bsd-EF S>Luc2(ns):T2A:TurboGFP vector (VectorBuilder, Inc.), without any additional transfection reagent. The MAF737 cell line was seeded $(50,000$ cells $)$ in a low attachment 96 -well plate (cat. no. 7007; Costar; Corning, Inc.) in 112.5 $\mu$ l Opti-MEM (cat. no. 31985-070; Gibco; Thermo Fisher Scientific, Inc.) without any growth factor or antibiotic. A total of $12.5 \mu 1$ virus was added on the same day. The cells were monitored for $48 \mathrm{~h}$, then centrifuged at $300 \mathrm{x} \mathrm{g}$ for $5 \mathrm{~min}$ at room temperature, and the cells were subsequently cultured at $37^{\circ} \mathrm{C}$ for $48 \mathrm{~h}$ in $75 \mathrm{~cm}$ tissue culture ventilated cap flask (Santa Cruz Biotechnology, Inc.) in RPMI medium (cat. no. 11875-093; Gibco; Thermo Fisher Scientific, Inc.). GFP signal was analyzed using a light Olympus microscope (CKX41) with fluorescent filters, at $\mathrm{x} 20$ and $\mathrm{x} 40$ magnification. Puromycin $(1 \mu \mathrm{g} / \mathrm{ml})$ was used for $48 \mathrm{~h}$ for antibiotic selection. Then, the cells were collected, $30 \mathrm{mg} / \mathrm{ml}$ D-luciferin potassium salt solution (Gold Biotechnology, Inc.) was added to the cells $(100,000)$ and luciferase expressing signal was analyzed using Xenogen IVIS 200 in vivo Imaging System (PerkinElmer, Inc.). Cells, without luciferase expressing signal, were used to exclude background and for normalization. The duration between transfection and activity measurement was 6 days.

Colony formation assay. The assays were performed as previously described in triplicate (25). The BT16 and MAF737 ATRT cell lines were seeded in 6-well plates, in triplicate, at a density of 500 cells/well, treated with Nutlin $(500,800$ and $1,000 \mathrm{nM})$ and idasanutlin $(1,10,50$ and $100 \mathrm{nM})$ and cultured for 10 days. The cells were stained with $0.25 \%$ crystal violet in methanol for $15 \mathrm{~min}$ at room temperature. Crystal violet-positive colonies ( $>50$ cells per colony) were counted using a Precise Electronic Counter (Heathrow Scientific, LLC) and a light inverted microscope at x2 magnification (Olympus S751; Olympus Corporation) (25). $\mathrm{IC}_{50}$ was calculated by comparing the colony numbers in treated and untreated cells.

Methylcellulose assay. The assay was performed as previously described (32). Briefly, the CHB-ATRT1 cell line wascultured for 10 days in 1:1 mixture $2.6 \%$ methylcellulose and complete growth (Neurobasal; Gibco; Thermo Fisher Scientific, Inc.) medium. Then, the colonies were stained with nitrotetrazolium blue chloride for $12 \mathrm{~h}$ at $37^{\circ} \mathrm{C}$ (cat. no. 6876; Sigma-Aldrich; Merck KGaA) and counted using a Precise Electronic Counter (Heathrow Scientific, LLC) and a light inverted microscope at x2 magnification (Olympus S751; Olympus Corporation).

Cell viability assay. The NHA, BT16, MAF737, CHLA04 and CHB ATRT cell lines were seeded in triplicate, at 50,000 cells/well, in 24-well plates, followed by DMSO or idasanutlin (30 and $300 \mathrm{nM}$, and 1 and $5 \mu \mathrm{M}$ ) treatment $24 \mathrm{~h}$ later. Then, the cells were trypsinized, collected, centrifuged at $300 \mathrm{x} \mathrm{g}$ for $5 \mathrm{~min}$ at room temperature and resuspended with ViaCount Reagent (cat. no. 4000-040; Luminex; Thermo Fisher Scientific, Inc.), $72 \mathrm{~h}$ later. The cells were counted using a Guava EasyCyte 8HT Flow Cytometry System (MilliporeSigma) and analyzed using Gyava Cytosoft Software version 5.3 (MilliporeSigma).

Western blot analysis. The protein expression levels were determined using western blotting analysis as previously described (33). The BT16 and CHB ATRT1 cells were lysed in RIPA buffer (cat. no. 89901; Thermo Fisher Scientific, Inc.), containing EDTA-free protease inhibitor (Roche Diagnostics). Protein concentrations were determined using a BCA Protein Assay Kit (Pierce; Thermo Fisher Scientific, Inc.). Protein (30 $\mu \mathrm{g}$ in total) was separated using 4-20\% SDS-PAGE (cat. no. 4561094; Bio-Rad Laboratories, Inc.). The membrane was blocked with $5 \%$ skimmed milk in TBS (Bio-Rad Laboratories, Inc.) with 1\% Tween-20, then incubated with primary antibodies in blocking solution for $12 \mathrm{~h}$ at $4^{\circ} \mathrm{C}$. The secondary antibodies $(\alpha$-mouse-HRP, cat. no. 7076; $\alpha$-rabbit-HRP, cat. no. 7074) (both diluted to 1:5,000) (both from Cell Signaling Technology, Inc.), were incubated with the membranes for $1 \mathrm{~h}$ at room temperature. The blots were developed with Immobilon Forte Western HRP Substrate (cat. no. WBLUF0500; MilliporeSigma) and imaged using Syngene GBox Chemi-SL1.4 gel documentation system. The following primary antibodies were used: TP53 (cat. no. 179477; Abcam), MDM2 (cat. no. 86934; Cell Signaling Technology, 
Inc.) p21 (cat. no. 2947; Cell Signaling Technology, Inc.), PUMA (cat. no. 12450; Cell Signaling Technology, Inc.) BAX (cat. no. 2772; Cell Signaling Technology, Inc.) Tubulin (cat. no. 3873; Cell Signaling Technology, Inc.) and $\beta$-actin (cat. no. 12262; Cell Signaling Technology, Inc.) (all 1:1,000).

In vivo xenograft models. An in vivo xenograft model was established, as previously described (25). A total of 10 female athymic nude (Foxn1 nu) mice (6-weeks-old; weight, 18-20 g) from Harlan Laboratories, were anesthetized with isoflurane (induction, 4\%; maintenance, 1.5-2\%; VetOne; MWI Boise) and immobilized in a stereotaxic apparatus (David Kopf Instruments). A small burr hole was drilled into the skull, at $1.5 \mathrm{~mm}$ lateral and $2.0 \mathrm{~mm}$ posterior to lambda, using a Dremel drill (Model 300; Dremel Manufacturing Company) and a $1.00 \mathrm{~mm}$ dental drill bit (cat. no. 514554; Stoelting Co.). Luciferase-expressing MAF737 ATRT cells (300,000 cells in $3 \mu \mathrm{l}$ serum-free medium) were injected $3.0 \mathrm{~mm}$ below the surface of the skull into the cerebellum, at a rate of $400 \mathrm{nl} / \mathrm{min}$, using a MicroUltra Pump (World Precision Instruments, Inc.), fitted with a $10 \mu \mathrm{l}$ Hamiliton syringe and a $26 \mathrm{G}$ needle (Hamilton Company). The burr hole was then sealed with bone wax (World Precision Instruments, Inc.), and the incision closed with sterile absorbable suture (Ethicon, Inc.). Carprofen ( $5 \mathrm{mg} / \mathrm{kg}$ ) was administered subcutaneously after the surgery, once daily, for 3 days (the day of surgery, and 24 and $48 \mathrm{~h}$ later). After the tumor was established in the cerebellum, the animals were randomized into 2 groups ( 5 mice per group): Control, vehicle and idasanutlin ( $30 \mathrm{mg} / \mathrm{kg} ; 5$ days per week for 2 weeks by oral gavage). The animals in the control group were treated with vehicle solution, containing 5\% DMSO (Sigma-Aldrich; Merck KGaA), 30\% polyethylene glycol (MilliporeSigma), 5\% Tween-80 (Sigma-Aldrich; Merck KGaA) in distilled water.

For bioluminescence analysis, D-luciferin potassium salt solution $(10 \mu \mathrm{l} / \mathrm{g}$ from a stock solution of $15 \mathrm{mg} / \mathrm{ml}$; Gold Biotechnology, inc.) was injected, via intraperitoneal injection and imaged using the Xenogen IVIS 200 in vivo Imaging System (PerkinElmer, Inc.). Tumor bioluminescence was analyzed using the Living Image v2.60.1 software (Caliper Life Sciences; PerkinElmer, Inc.). The body weight of all the mice was measured weekly (24) and mice were monitored daily and euthanized when they reached the endpoint of the experiment ( $>15 \%$ loss in body weight, irreversible neurogical effect or inability to eat or drink). The mice were kept at $21-23^{\circ} \mathrm{C}$, with $30-50 \%$ humidity and $14 \mathrm{~h}$ light/10 h dark cycle, with hyperchlorinated water and fed a standard Envigo diet (irradiated, x2,920). The animals were euthanized with $\mathrm{CO}_{2}$ followed by cervical dislocation. The flow rate of $\mathrm{CO}_{2}$ was $30 \%$ of the displacement volume/min. All Animal procedures were performed in accordance with the National Research Council's Guide for the Care and Use of Laboratory Animals and were approved by the University of Colorado Anschutz Campus Institutional Animal Care and Use Committee (approval number, 00052).

MRI. The MRI images were obtained using a Bruker 9.4 Tesla BioSpec MRI Scanner (Bruker Corporation), as previously described (27). T2-turboRARE images of the sagittal and axial panels were acquired and analyzed using Bruker
ParaVision NEO360 v2.0 software (Bruker Corporation). A region of interest was manually segmented on each anatomic slice and tumor volume in $\mathrm{mm}^{3}$ was calculated by a radiologist. The apparent diffusion coefficients (ADC; $\mathrm{s} / \mathrm{mm}^{2}$ ) were calculated from diffusion-weighted imaging maps, as a criterion for tumor cellularity.

Immunohistochemistry. Tissue from the patient samples or experimental animals was fixed in $10 \%$ formalin for 3 days at room temperature and submitted to the University of Colorado Denver Tissue Histology Shared Resource for sectioning and staining (25). Paraffin-embedded sections (5- $\mu \mathrm{m})$ were prepared for immunodetection of Ki67 (1:500; cat. no. RM-9106; Thermo Fisher Scientific, Inc.), MDM2 (1:50; cat. no. LS-C199239; LifeSpan Bioscience, Inc.), P53 (1:50; cat. no. 2527; Cell Signaling Technology, Inc.) and Caspase3 (1:1,000; cat. no. 9661; Cell Signaling Technology, Inc.). The antigens were revealed in BORG solution ( $\mathrm{pH} 9.5)$ for $10 \mathrm{~min}$ at $110^{\circ} \mathrm{C}(\mathrm{NxGen}$ Decloaker chamber) (both from Nobel Biocare Services AG) with a $10 \mathrm{~min}$ ambient cool down. Immunodetection was performed using a Benchmark XT autostainer for $32 \mathrm{~min}$ at $37^{\circ} \mathrm{C}$ using a modified I-VIEW DAB detection system (both from Ventana Medical Systems, Inc.; Roche Diagnositics). Endogenous peroxidase was blocked with $3 \%$ hydrogen peroxide for $4 \mathrm{~min}$. The secondary antibody cocktail from the I-VIEW DAB kit (goat anti-mouse and goat anti-rabbit; prediluted; cat. no. 5266157001; Ventana Medical Systems, Inc.) was removed and replaced with full strength rabbit ImmPress polymer (one step polymer goat anti-rabbit conjugated to HRP; cat. no. MP-7401; Maravai LifeSciences). The streptavidin-HRP conjugated enzyme from the I-VIEW DAB kit (cat. no. 5266157001, Ventana Medical Systems, Inc.) was then added and replaced with diluted rabbit ImmPress polymer (1:2 dilution in PBS pH 7.6; cat. no. MP-7401; Maravai LifeSciences). Secondary and enzyme incubations were performed at $8 \mathrm{~min}$ each at $37^{\circ} \mathrm{C}$ according to standard protocol dictated by the I-VIEW kit. DAB was used to visualize the antigen-antibody complexes using a standard protocol. All the sections were counterstained with hematoxylin for $2 \mathrm{~min}$ at $37^{\circ} \mathrm{C}$, blued for $10 \mathrm{sec}$ at $37^{\circ} \mathrm{C}$ in $1 \%$ ammonium hydroxide, dehydrated in a descending alcohol series, cleared in xylene and mounted using synthetic resin (Cancer Diagnostics, Inc.) with a coverglass at room temperature. Negative controls were used to confirm the specificity of immunostaining included omission of the primary antibody incubation step and substitution of the primary antibody diluent. The images were captured using a BZ-X710 all-in-one fluorescence microscope (Keyence Corporation) and quantified with BZ-X viewer v.01.03.01.01 (Keyence Corporation).

Immunofluorescence. The BT16 ATRT cell line (3,000 cells) were seeded in poly-D-lysine-coated chamber slides. The next day, the cells were treated with $30 \mathrm{nM}\left(\mathrm{IC}_{50} ; 72 \mathrm{~h}\right)$, idasnutlin ( $\mathrm{IC}_{50}$, detected from colony formation assay) or DMSO. The cells were radiated with $4 \mathrm{~Gy}$ radiation, $72 \mathrm{~h}$ later. Then, the cells were washed with PBS (cat. no. 21-040-CV; Corning, Inc.), fixed for $15 \mathrm{~min}$ at room temperature with $4 \%$ paraformaldehyde (cat. no. J61899; Alfa Aesar; Thermo Fisher Scientific, Inc.), blocked for $30 \mathrm{~min}$ at room temperature in 5\% skimmed milk and 0.05\% TritonX-100 (cat. no. 93443; Sigma-Aldrich; Merck KGaA) in PBS and incubated with the 
primary antibody for $12 \mathrm{~h}$ at $4^{\circ} \mathrm{C}$ and the secondary antibodies for $1 \mathrm{~h}$ at room temperature, as previously described (25). The following antibodies were used: $\gamma \mathrm{H} 2 \mathrm{AX}$ (1:300; cat. no. 9718; Cell Signaling Technology, Inc.), Alexa Fluor 488-conjugated secondary antibody (1:500; cat. no. 560445; BD Pharmingen; BD Biosciences). Then, the cells were washed with $0.05 \%$ TritonX-100 in PBS 3 times, for 5 min each time and mounted with ProLong Gold antifade reagent containing DAPI (cat. no. 36935; Sigma-Aldrich; Merck KGaA) (25). The images were captured using a fluorescence microscope (BZ-X700; Keyence Corporation), at x40 magnification.

Irradiation of the culture cells. A JL Shepherd Model 81-14 irradiator, with $137 \mathrm{Cs}$ source, was used to radiate cells. The dose of radiation, from the $137 \mathrm{Cs}$ source was $1.09 \mathrm{~Gy} / \mathrm{min}$. The BT16 cell line was radiated with $2,4,6,8$ and $10 \mathrm{~Gy}$ for $1.8,3.6,5.5,7.5$ and $9.7 \mathrm{~min}$, respectively.

Combination of idasanutlin and ionizing radiation. For the combination of idasanutlin treatment and ionizing radiation, the BT16 cell line was seeded at different densities (500, $1,000,2,000,4,000,6,000$ and 8,000 cells/per well) in a 6 -well plate in triplicate for $24 \mathrm{~h}$ before the addition of $18 \mathrm{nM}$ idasanutlin ( $\mathrm{IC}_{30}$ from previous calculation) or DMSO. The cells were treated to idasanutlin for $6 \mathrm{~h}$, then drug-containing medium was aspirated and normal culture medium (RPMI, supplemented with $10 \%$ FBS and $1 \%$ Pen/Strep) was added. The cells were then immediately irradiated with $2,4,6,8$ or $10 \mathrm{~Gy}$. After 10 days of additional growth, the cells were stained with $0.25 \%$ crystal violet in methanol for $15 \mathrm{~min}$ at room temperature. Crystal violet-positive colonies ( $>50$ cells per colony) were counted using a Precise Electronic Counter (Heathrow Scientific, LLC) and a light inverted microscope (Olympus S751; Olympus Corporation). The survival curves were generated after normalizing to idasanutlin treatment-induced death. Non-linear regressions were calculated. The radiation dose intersecting the non-linear regression for a $10 \%\left(\mathrm{SF}_{0.1}\right)$ and $50 \%\left(\mathrm{SF}_{0.5}\right)$ surviving fraction was calculated for drug dose. The sensitizer enhancement ratio (SER) was then calculated as follows: $\mathrm{SER}=\mathrm{SF}_{\mathrm{x}} \mathrm{DMSO} / \mathrm{SF}_{\mathrm{x}} \mathrm{X}$ nM idasanutlin. The SER allows for direct comparison of the effect of the putative sensitizing agent relative to control treated cells.

Flow cytometry assay. The BT16 cell line was seeded in $10 \mathrm{~cm}$ plates $\left(1 \times 10^{6}\right.$ cells/well). The cells were treated the following day with idasanutlin $\left(\mathrm{IC}_{50}, 30 \mathrm{nM} ; 72 \mathrm{~h}\right.$ ), or DMSO for $72 \mathrm{~h}$, with or without 4 Gy radiation, then fixed with $4 \%$ formaldehyde for $15 \mathrm{~min}$ at room temperature. The fixed cells were then washed with 3\% FBS (Atlanta Biologicals, Inc.) in PBS and permeabilized with methanol on ice for $10 \mathrm{~min}$. The cells were incubated with a $\gamma \mathrm{H} 2 \mathrm{AX}$ (1:20; cat. no. 562377; BD Pharmingen; BD Biosciences) antibody for $1 \mathrm{~h}$ at room temperature. Flow Cytometry Analysis was performed using an Amnis FlowSight Flow Cytometer (MilliporeSigma) and analyzed with IDEAS software v6.1 (Luminex Corporation). Unstained cells were used for gating to obtain the percentage of positive $\gamma \mathrm{H} 2 \mathrm{AX}$ cells.

Measurement of mitochondrial reactive oxygen species (ROS) using flow cytometry. MitoSOX-based flow cytometry was used to measure the mitochondrial ROS, as previously described (34). Briefly, the BT16 cell line was treated with DMSO or idasanutlin $\left(\mathrm{IC}_{50}, 30 \mathrm{nM} ; 2 \mathrm{~h}\right)$, then with 4 Gy radiation for $24 \mathrm{~h}$. The cells were trypsinized and 500,000 cells from each condition were centrifuged $(300 \mathrm{x} g$ for $5 \mathrm{~min}$ at room temperature) and resuspended in sterile $1 \mathrm{X}$ PBS, containing $1 \mathrm{mM}$ MitoSOX Red mitochondrialsuperoxide indicator (cat. no. M36008; Invitrogen; Thermo Fisher Scientific, Inc.). The samples were then placed on a shaking water bath $\left(37^{\circ} \mathrm{C}\right)$ for $30 \mathrm{~min}$ and protected from light, washed three times with PBS, then resuspended in cell staining buffer (BioLegend, Inc.) containing DAPI (1:10,000 dilution from $1 \mathrm{mg} / \mathrm{ml}$ stock concentration). Finally, the samples were analyzed using a CytoFlex LX flow cytometer (Beckman Coulter, Inc.) to measure the mean fluorescence intensity and the percentage of stained cells on DAPI-(live) cells. The results are represented as the cell count from the cell fluorescence emission, representing the size of the cell population emitting the red fluorescence, and as the mean fluorescence intensity of MitoSOX across different treatment conditions. The results were analyzed using CytExpert, v2.4.028 (Beckman Coulter, Inc.).

Statistical analysis. Statistical analysis was performed using GraphPad Prism v8 software (GraphPad Software, Inc.). Either one- or two way ANOVA, or one- or two-tailed Student's t-test (unpaired) was used for comparisons between groups. Kruskal-Wallis test was used to analyze gene expression between normal cerebellum samples and each ATRT subtype. Kaplan-Meier survival curve comparisons were performed using log-rank (Mantel-Cox) test. $\mathrm{P}<0.05$ was considered to indicate a statistically significant difference. The data are presented as the mean \pm standard error of the mean. The experiments were repeated indepently three times. The effect of TP53 knockdown in genome-wide knockdown screens was performed using DepMap (DepMap.org).

\section{Results}

P53-MDM2 is a therapeutic target in ATRT. To systematically identify genes that could be therapeutic targets in SMARCB1 deleted ATRT, a shRNA screen was performed to targeting 408 genes associated with transcription- and chromatin-related processes. A patient derived SMARCB1 deleted ATRT cell line (MAF737) was transduced with a pooled lentiviral shRNA library, consisting of 4-10 shRNAs per gene $(4,180$ total shRNAs). A total of 408 genes were analyzed. Genomic DNA was isolated from the transduced cells 4 and 21 days after transduction and sequenced with an Illumina Hiseq instrument The unique shRNA abundance was compared between the samples collected on day 21 vs. day 4 . The differential enrichment of shRNAs targeting genes was assessed using R-based package DESeq2. The fold change in the genes enriched on day 21 vs. day 4 are shown in Fig. 1A. High confident candidate genes were defined as those with log fold change $\geq 2$, with $\geq 2$ shRNAs per gene with adjusted $P<0.05$. Genes targeted by shRNA that enhanced tumor cell growth were analyzed, as this suggested tumor suppressive activity (Fig. 1A). Among the key druggable genes that enhanced ATRT cell growth following knockdown of expression was 
A
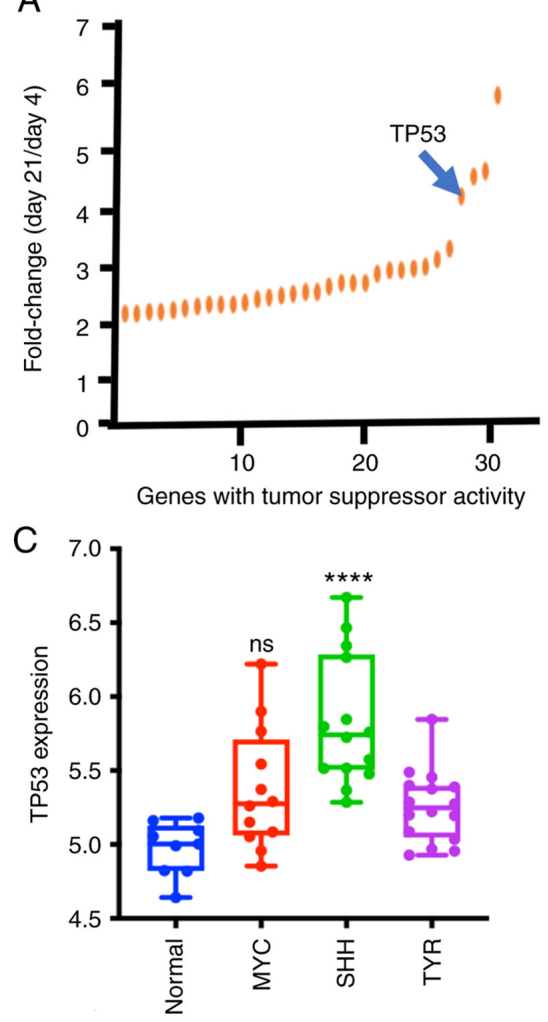

$\mathrm{E}$

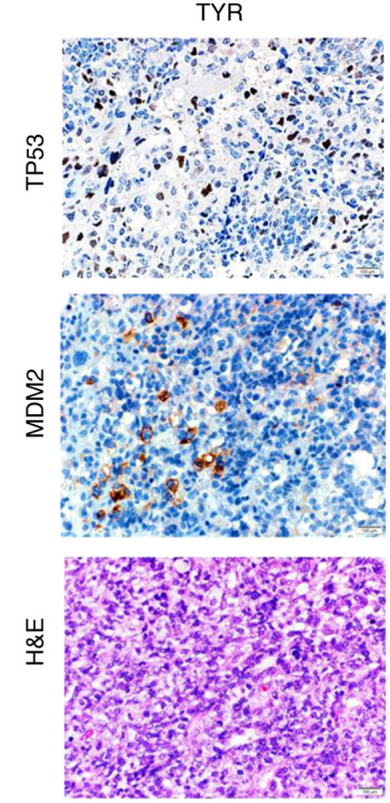

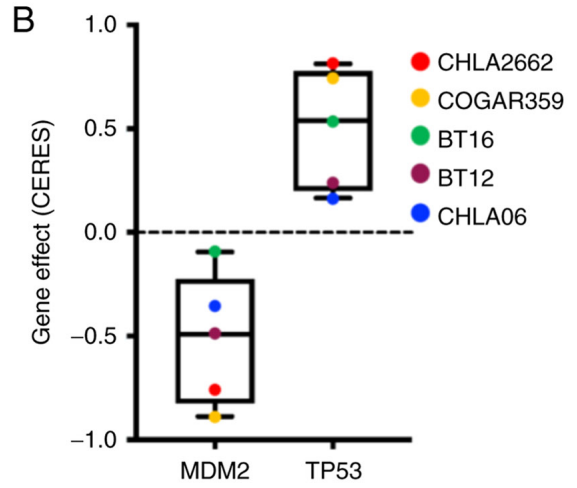

D 15.2$]$

$\mathrm{SHH}$
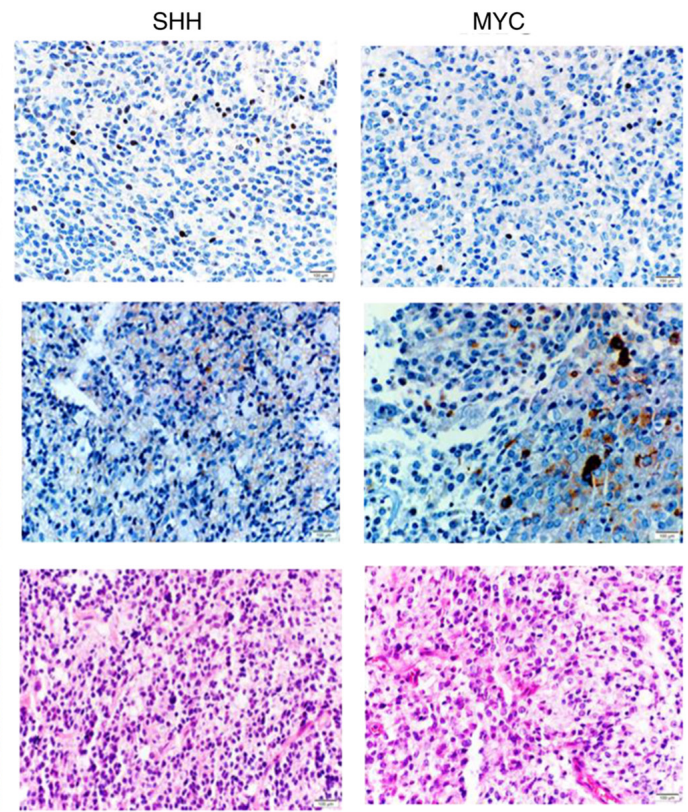

Figure 1. Identification of mediators of ATRT cell growth and their expression level in the three ATRT subgroups. (A) shRNA screening revealed that TP53 knockdown enhanced ATRT cell growth. (B) TP53 and MDM2 dependency was analyzed using the Dependency Map portal. TP53 was shown to be a key dependency in BT16 ATRT. A DepMap negative score for CRISPR demonstrates high dependency of ATRT from MDM2. mRNA expression level of (C) TP53 and (D) MDM2 in tumors from patients with ATRT from the three subgroups (TYR, SHH and MYC) as compared with that in the normal brain and between groups, using the GSE70678 dataset. Error bars represent standard error of the mean. ${ }^{* * *} \mathrm{P}<0.01 ;{ }^{* * *} \mathrm{P}<0.001 ;{ }^{* * * *} \mathrm{P}<0.0001$ vs. normal tissue. (E) Immunohistochemistry staining of TP53, MDM2 and H\&E in tumors from patient with ATRT from different ATRT subgroups. x40 magnification. ATRT, atypical teratoid rhabdoid tumor; ns, not significant.

TP53 (Fig. 1A). To confirm the results, the effect of TP53 knockdown in genome-widescreens was examined using DepMap (DepMap.org (35). The DEMETER2 dependency score is based on data from a cell depletion assay. A lower DEMETER2 score indicates a higher likelihood that the gene is essential in a given cell line. A score of 0 indicates a gene is not essential. A positive score reflects a progrowth phenotype. TP53 was shown to be a key gene in BT16 (Figs. 1B and S1A). As TP53 activity is regulated by MDM2, the dependency of ATRT cells on MDM2 was also analyzed. A DepMap negative score for CRISPR demonstrates high dependency of ATRT from MDM2. To evaluate the importance of the 
A
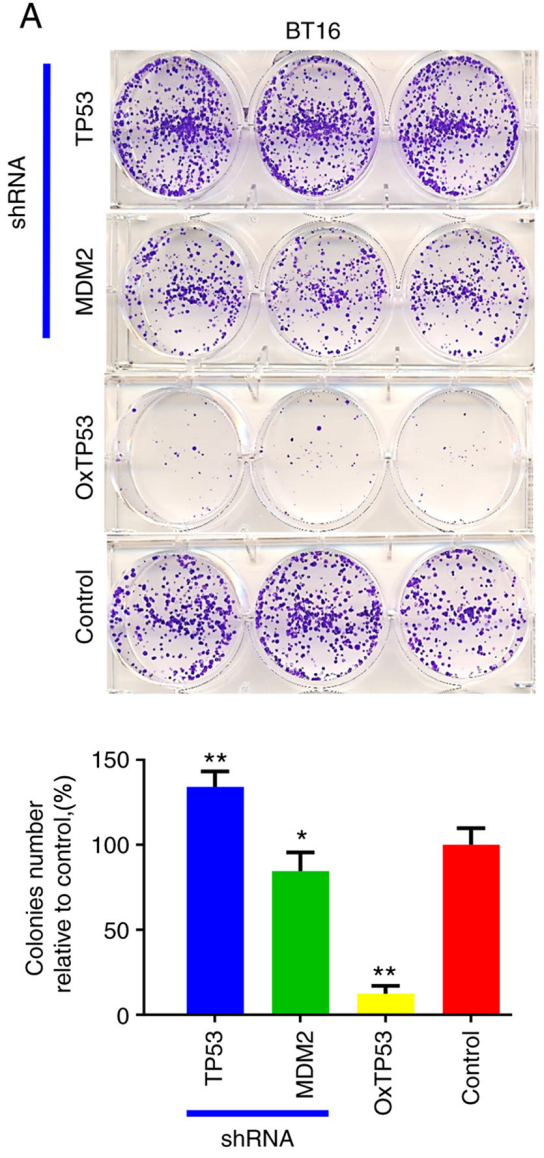

C

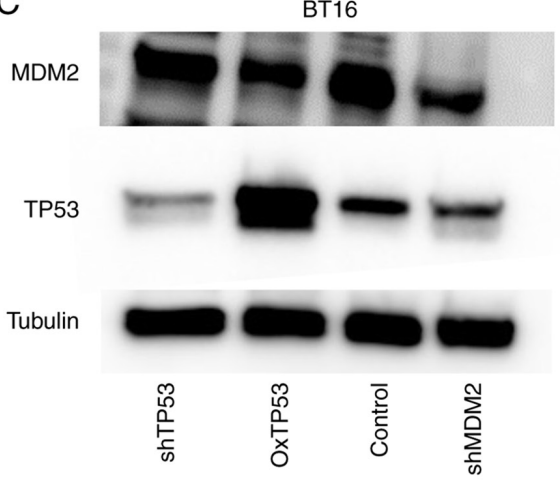

B
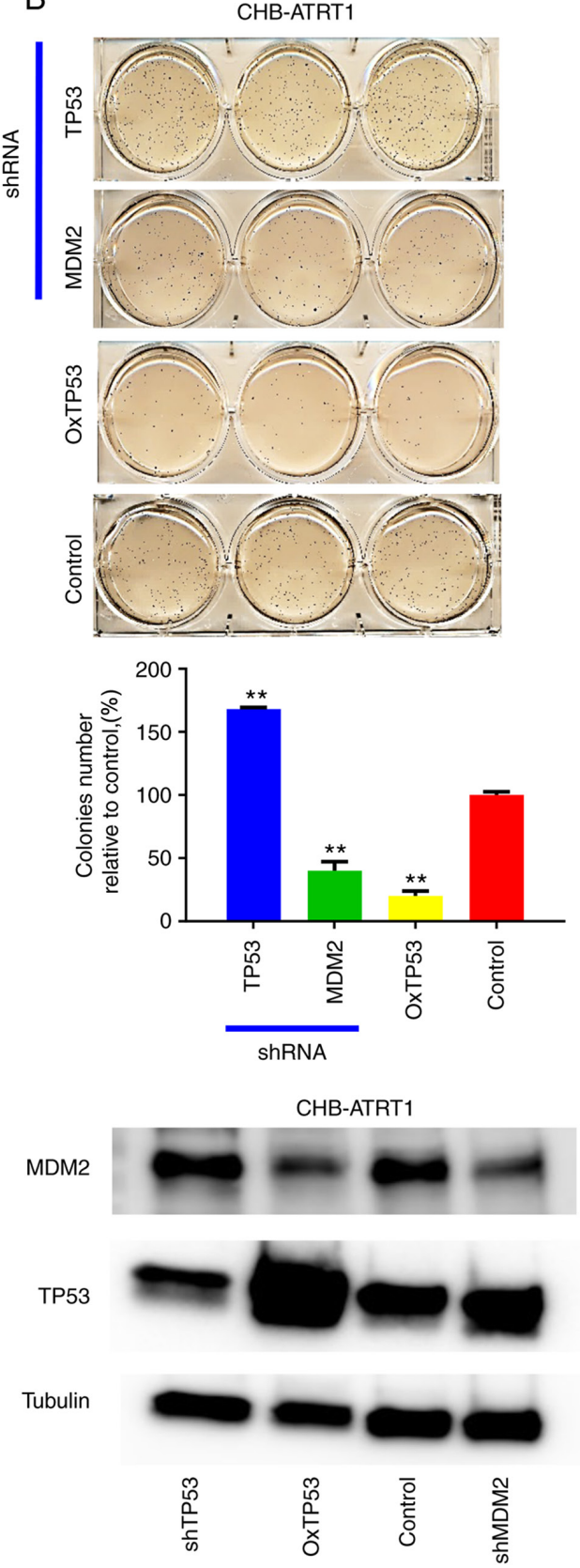

Figure 2. Genetic knockdown and activation of TP53 in the ATRT cell lines. Colony formation assay was performed in the (A) BT16 and (B) CHB-ATRT1 cell lines following transfected with either TP53 or MDM2 shRNAs and with overexpression of TP53. The results were also quantified. ${ }^{*} \mathrm{P}<0.05 ;{ }^{* * *} \mathrm{P}<0.01$ vs. control. (C) Western blot analysis was performed in the BT16 and CHB-ATRT1 cell lines following transfection with TP53 or MDM2 shRNAs and with overexpression of TP53. ATRT, atypical teratoid rhabdoid tumor; sh, short hairpin; Ox, overexpression.

TP53/MDM2 axis in ATRT, the mRNA expression level of TP53 and MDM2 was analyzed in a cohort of 42 ATRT samples, from all subgroups (11). A total of 14 samples were ATRT/SHH, 15 samples were ATRT/TYR, 13 samples were ATRT/MYC and 9 samples were normal cerebellum (Fig. 1C). TP53 mRNA expression level was heterogeneous in ATRT compared with that in the normal cerebellum $(\mathrm{P}=0.057)$ (Fig. 1C). On the other hand, MDM2 mRNA expression level was significantly elevated in all ATRT samples as compared with that in the normal brain $(\mathrm{P}<0.01$; Fig. 1D). The highest level of MDM2 expression was in the ATRT/TYR subgroup $(\mathrm{P}<0.001$; Fig. 1D). To further confirm the expression level of TP53 and MDM2 in ATRT, the patient samples were analyzed using immunohistochemistry. TP53 was variably expressed in all ATRT subgroups, whereas MDM2 was significantly expressed in the TYR and MYC subgroups. H\&E stain confirms ATRT tumors (Figs. 1E and S1B).

P53 and MDM2 are critical for ATRT cell proliferation and clonogenicity in vitro. We hypothesized that ATRT cell growth is driven by overexpression of MDM2. Consequently, the inhibition of MDM2 would hinder ATRT cell growth. The hypothesis was investigated using shRNA to knockdown the expression level of MDM2 in the BT16 (ATRT/MYC) and CHB-ATRT1 (ATRT/MYC) cell lines (Fig. 2). Using a colony formation assay, in both the BT16 and CHB-ATRT1 cell lines, transfection with 
A
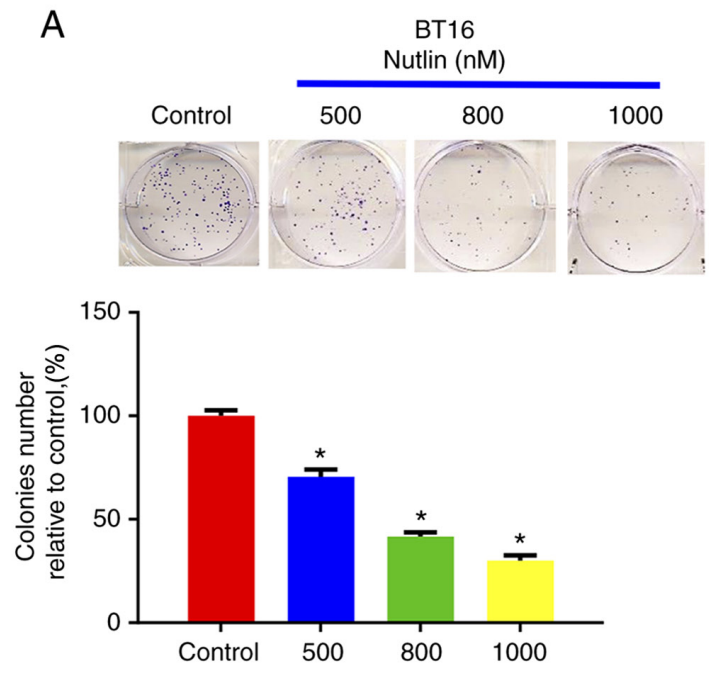

C
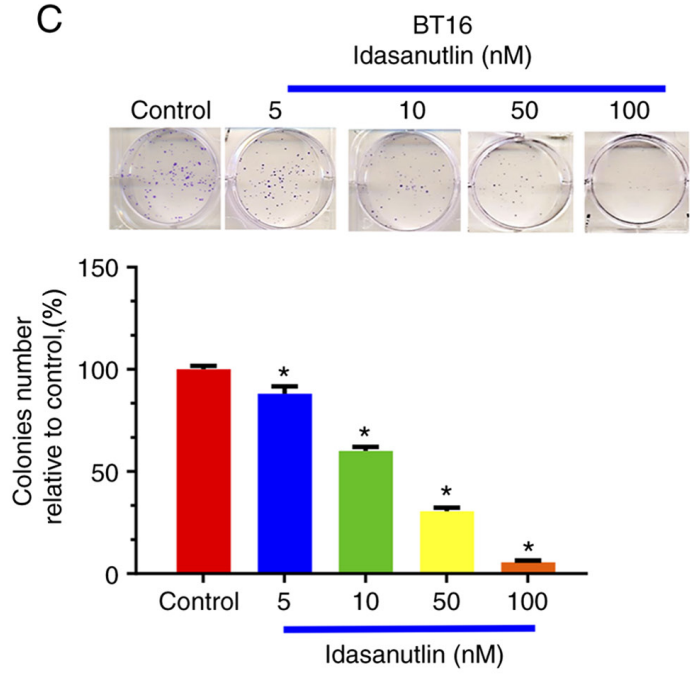

B
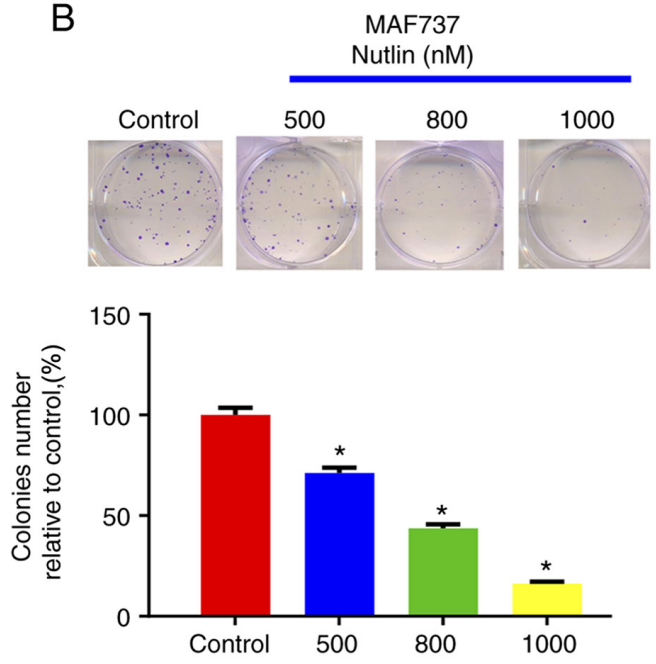

$\mathrm{D}$
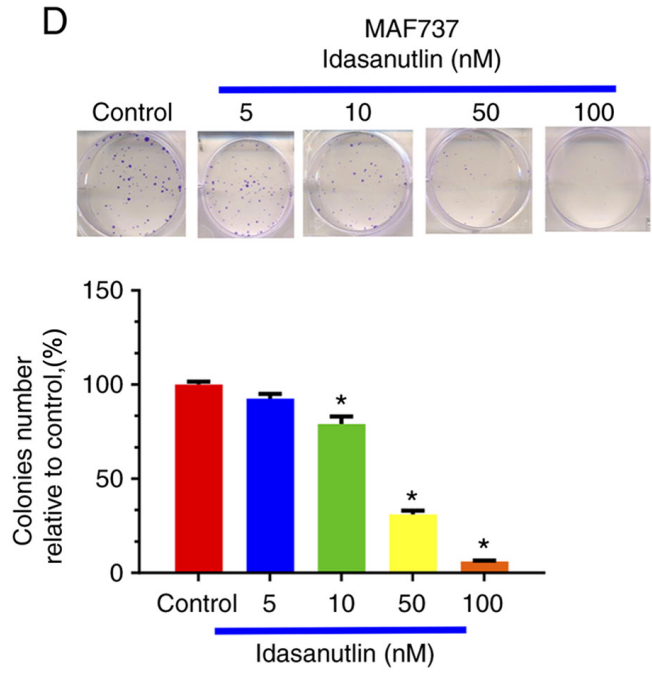

Figure 3. Chemical inhibition of MDM2 using Nutlin suppresses ATRT growth in vitro. Nutlin3 inhibits colony formation ability in the (A) BT16 and (B) MAF737 ATRT cell lines. The results were subsequently quantified. * $\mathrm{P}<0.05$ vs. control. (C) The MDM2 inhibitor, idasanultin reduced the colony formation ability in the (C) BT16 and (D) MAF737 cells lines. The results were subsequently quantified. * $\mathrm{P}<0.05$ vs. control. The error bars show the standard error of the mean. ATRT, atypical teratoid rhabdoid tumor.

shMDM2 significantly decreased the number of colonies formed after 10 days for the BT16 (P<0.05; Fig. 2A) and CHB-ATRT1 ( $\mathrm{P}<0.01$; Fig. 2B) cell lines. Conversely, shTP53 enhanced ATRT cell growth $(\mathrm{P}<0.01$; Fig. $2 \mathrm{~A}$ and $\mathrm{B})$. Overexpression of TP53 suppressed colony formation in the BT16 and CHB-ATRT1 ATRT cell lines ( $\mathrm{P}<0.01$; Fig. $2 \mathrm{~A}$ and $\mathrm{B})$. Knockdown and overexpression of MDM2 and TP53 was verified using western blot analysis (Figs. 2C, S2 and S3). These data suggest that activation of TP53 maybe a potential therapeutic target in ATRT.

Chemical inhibition of MDM2 suppresses ATRT growth in vitro. To further evaluate the effect of MDM2 inhibition on ATRT cells, Nutlin3, a potent and selective MDM2 antagonist was subsequently used. Nutlin3 potently inhibited the colony formation ability of both the BT16 and MAF737 cell lines, in a dose-dependent manner, with $\mathrm{IC}_{50}, 750$ and $700 \mathrm{nM}$ for the BT16 and MAF737 cell lines, respectively (Figs. 3A and B, and $\mathrm{S} 4 \mathrm{~A}$ and $\mathrm{B})$.

Nutlin was the initial inhibitor identified that targeted the interaction of MDM2 with TP53 (18) Idasanutlin (RG7388), a second-generation Nutlin, with improved potency, toxicity, selectivity and bioavailability is in clinical development (19). Therefore, the ability of idasanutlin to inhibit the ATRT cell lines was investigated. Idasanutlin potently attenuated ATRT cell colonal formation, at a low nanomolar concentration, with an $\mathrm{IC}_{50}$ of $30 \mathrm{nM}$ (Figs. 3C and D, and S4C and D).

\section{Idasanutlin inhibits ATRT growth depending on MDM2} expression in subgroups. Next, the effect of chemical inhibition of MDM2, in additional cell lines from the different ATRT subgroups was analyzed. The CHLA04 and CHB-ATRT1 cell lines, from the SHH and MYC subgroups, respectively were chosen. First, the cells from different ATRT subgroups were treated with a small concentration of idasanutlin $(30 \mathrm{nM})$. Cell viability and $\mathrm{IC}_{50}$ measurements were performed using flow cytometry. The CHLA04 cell line was less sensitive compared with that in the BT16 and MAF737 cell lines (Fig. 4A). In addition, $30 \mathrm{nM}$ idasanutlin decreased the cell viability in the CHB-ATRT1; however, the result was not significantly different (Fig. 4A). Then, all the cell lines were treated with varying 
Table I. IC $_{50}$ values in different ATRT and normal brain cell lines following treatment with idasanutlin.

\begin{tabular}{|c|c|c|c|c|c|}
\hline \multirow[t]{2}{*}{ Variable } & \multicolumn{5}{|c|}{ ATRT subtype } \\
\hline & Control & TYR/MYC & TYR & $\mathrm{SHH}$ & MYC \\
\hline & Cell line & & & & \\
\hline & NHA & BT16 & MAF737 & CHLA04 & CHB-ATRT1 \\
\hline $\mathrm{IC}_{50}, \mathrm{nM}$ & NA & 30 & 30 & 140 & 1,500 \\
\hline
\end{tabular}

NHA, normal human astrocytes; ATRT, atypical teratoid rhabdoid tumor.
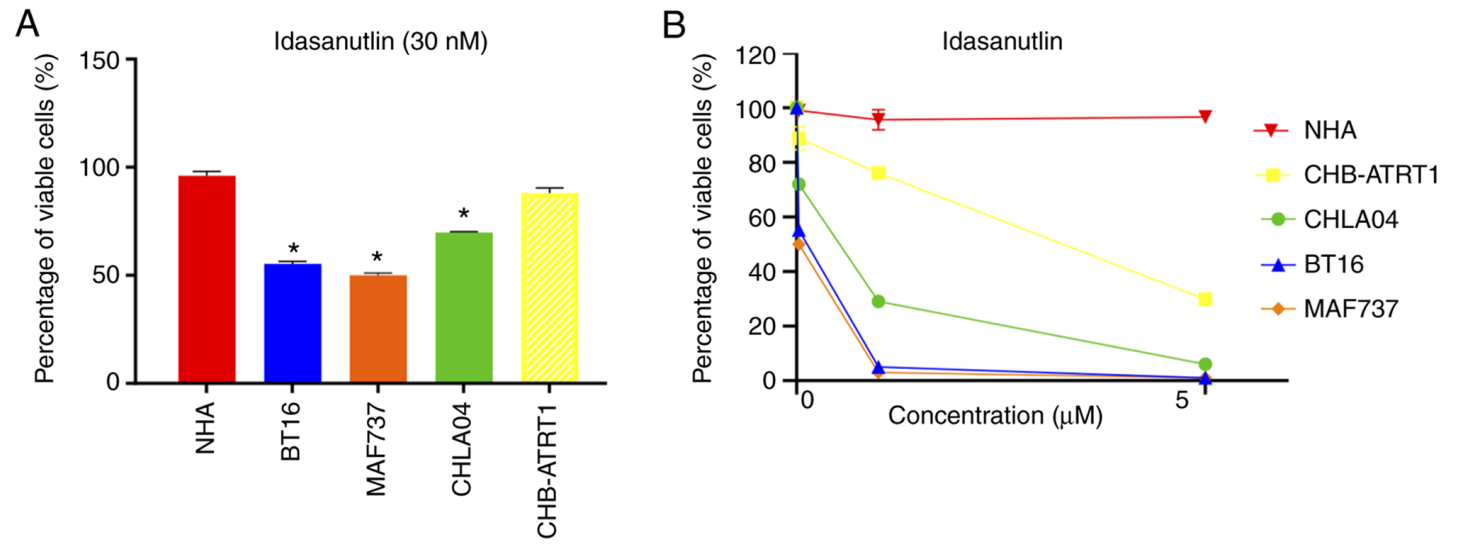

Figure 4. Treatment with idasanutlin in ATRT cell lines from different subgroups and NHA. ATRT cells from different subgroups (TYR, SHH and MYC) were treated with idasanutlin (A) at $30 \mathrm{nM}\left(\mathrm{IC}_{50}\right)$ and (B) different concentrations, then cell viability was measured. $\mathrm{n}=3$. The error bars show the standard error of the mean. ${ }^{*} \mathrm{P}<0.05$ vs NHA. NHA, normal human astrocytes; ATRT, atypical teratoid rhabdoid tumor.

concentrations of idasanutlin. The results revealed that the CHB-ATRT1 cell line was more resistant to idasanutlin treatment (Fig. 4B; Table I). The $\mathrm{IC}_{50}$ for the CHB-ATRT1/MYC cell line, with the lowest MDM2 expression level, increased as compared with that in the CHLA04/SHH, BT16/TYR/MYC and MAF737/TYR cell lines, with high MDM2 expression level (Fig. 4B; Table I). Furthermore, the NHA cell line was also treated with different concentrations of idasanutlin and the results showed that idasanutlin treatment, even at $5 \mu \mathrm{M}$ did not change cell viability (Fig. 4A and B).

Pharmacological inhibition of MDM2 drives a therapeutic effect in intracranial orthotopic xenograft models of ATRT. Subsequently, it was investigated whether MDM2 inhibition, by idasanutlin, could be effective in an intracranial orthotopic xenograft in vivo model. The MAF737 cell line was transduced with a luciferase expressing vector and stereotactically injected into the cerebellum of immunocompromised mice. The mice were randomized and treated with vehicle or idasanutlin $(30 \mathrm{mg} / \mathrm{kg})$ by oral gavage, 5 times per week for 2 weeks. The treatment response was determined using patient-analogous MRI. In the idasanutlin treated group, MRI images showed decreases in tumor growth and invasive tissue (Fig. 5A). Volumetric analysis of the tumors, using MRI, demonstrated a significantly smaller tumor size in treated animals compared with that in the control animals $(\mathrm{P}<0.05$; Fig. $5 \mathrm{~B})$. ADC was calculated from diffusion-weighted imaging maps, as a measure of tumor cellularity. After one week of treatment, ADC was significantly higher in the idasanutlin treated group compared with that in the vehicle group $(\mathrm{P}<0.05$; Fig. $5 \mathrm{C})$, which suggests that idasanutlin treatment leads to necrosis/apoptosis. The results showing that idasanutlin treatment resulted in higher ADC values compared with that in the control group further indicates that idasanutlin may be a promising target in a clinical setting. Histological evaluation confirmed diffusely infiltrating, SMARCB1 deleted tumors (Fig. 5D), with decreased proliferative index in Ki67 staining in idasanutlin treated animals ( $\mathrm{P}<0.05$; Fig. 5D and $\mathrm{E})$. Furthermore, analysis of tumor tissues revealed an increased number of caspase-3-positive cells in the idasanutlin treated group, further demonstrating induction of apoptosis in vivo $(\mathrm{P}<0.05$; Fig. 5D and $\mathrm{E})$. Tumor formation was analyzed using bioluminescence (Fig. 5G). Treatment with idasanutlin resulted in significantly decreased bioluminescent signal, suggesting attenuation of tumor growth $(\mathrm{P}<0.05$; Figs. $5 \mathrm{G}$ and $\mathrm{H}$, and $\mathrm{S} 5 \mathrm{~A})$. Furthermore, animals treated with idasanutlin survived longer compared with that in the control animals. Median survival time for mice treated with idasanutlin was 14 days vs. 9 days in mice treated with the vehicle. Kaplan-Meier survival analysis revealed an improvement in overall survival time in mice treated with idasanutlin comparied with those treated with vehicle ( $\mathrm{P}=0.18)$ (Fig. 5I). Idasanutlin was not toxic to the mice and did not lead to any weight lost as compared with that in the vehicle control group (Fig. S5B).

Idasnutlin enhances radiation sensitivity of the ATRT cells. Radiation is one of the main treatment methods in most 
A
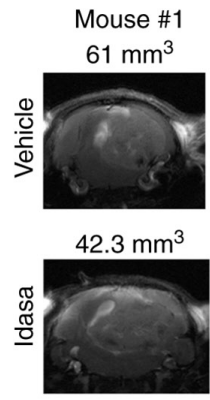

$\mathrm{D}$
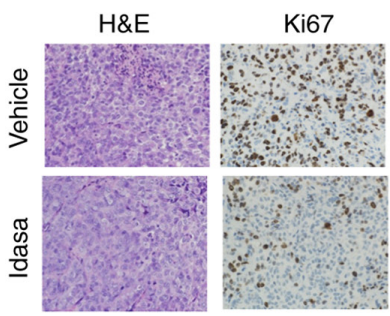

G

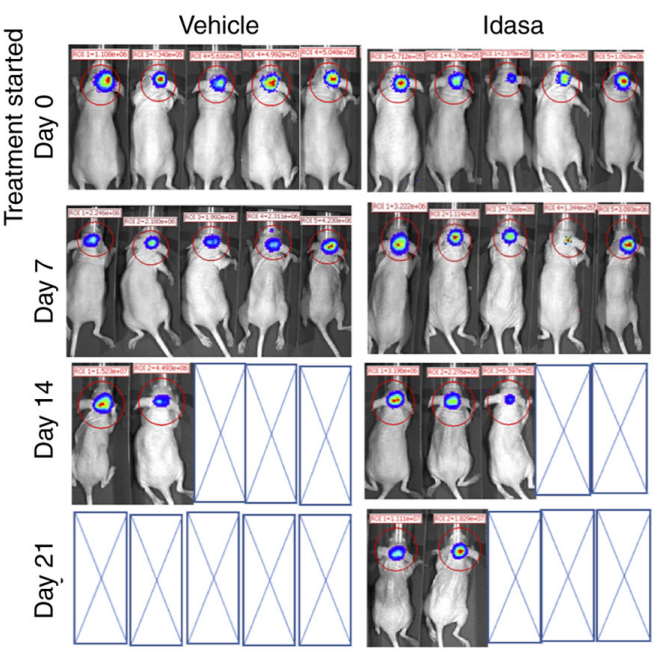

B

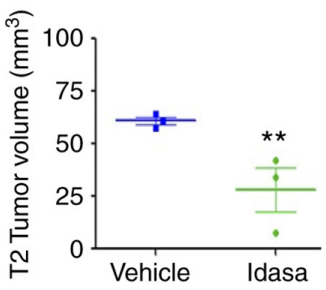

E

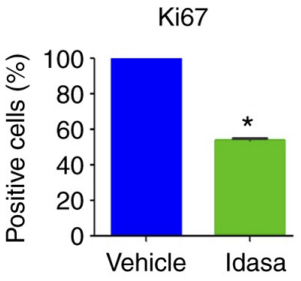

C

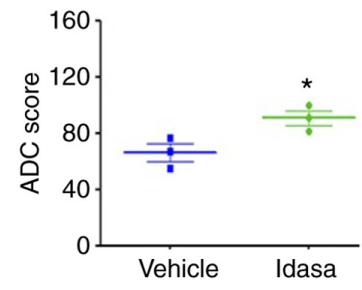

$\mathrm{F}$

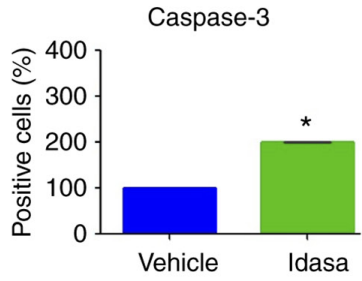

$\mathrm{H}$

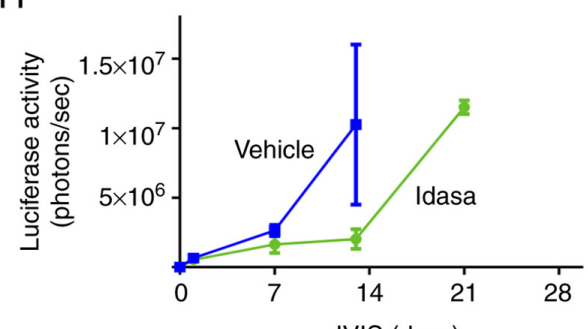

I

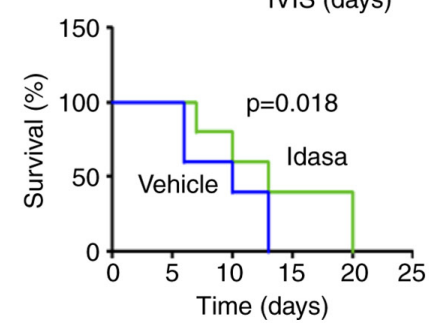

Figure 5. MDM2 inhibition using idasanutlin suppressed atypical teratoid rhabdoid tumor growth in vivo. (A) Representative axial T2-weighted turbo RARE MRI sequences of mice in the vehicle and idasanutlin treatment groups 1 week after treatment was started. (B) MRI volumetric analysis demonstrates decreased tumor volume in the idasanutlin treated cohort. $\mathrm{n}=3$. ${ }^{* *} \mathrm{P}<0.01$. (C) ADC was calculated from diffusion-weighted imaging maps, as a criterion for tumor cellularity. After one week of treatment, ADC was significantly higher in the idasanutlin treated group compared with that in the vehicle group. ${ }^{*} \mathrm{P}<0.05$. (D) H\&E, Ki67 and caspase-3 IHC images from the cerebellar tumors collected at the end-points of the in vivo xenograft model. x40 magnification. The results were subsequently quantified for (E) Ki67 and (F) caspase3. Plots show values from quantification of 3 representative images from 3 treated mice. " $\mathrm{P}<0.05$; ${ }^{* *} \mathrm{P}<0.01$. (G) IVIS images of mice on different days of treatments. Treatment started at day 0 and the results were subsequently $(\mathrm{H})$ quantified. The error bars show the standard error of the mean. (I) Survival analysis of idasanutlin vs. vehicle treated animals. Median survival times of idasanutlin treated animals was 14 days vs. 9 days in the vehicle treated group. Idasanutlin treatment slowed tumor growth and increased mouse survival. ADC, apparent diffusion coefficient; IHC, immunohistochemtistry; idasa, idasanutlin.

ATRT clinical protocols (2). To investigate whether inhibition of MDM2 could alter the sensitivity of the ATRT cells to radiation, the BT16 cell line was treated with $18 \mathrm{nM}$ idasanutlin $\left(\mathrm{IC}_{30}\right.$ as previously calculated) for $6 \mathrm{~h}$ before irradiation and the effects were evaluated using a colony formation assay (Fig. 6A). SER was calculated at the $10 \%$ $\left(\mathrm{SF}_{0.1}\right)$ and $50 \%\left(\mathrm{SF}_{0.5}\right)$ surviving fraction in idasanutlin and DMSO treated cells. For the BT16 cell line pretreated with idasanutlin, the SERs were 1.632 for $\mathrm{SF}_{0.1}$ and $\mathrm{SF}_{0.5}$ (Fig. 6B). The SER demonstrates the effect of the sensitizing agent relative to the control in the presence of radiation. A SER greater than one indicates a synergistic effect of the sensitization agent with radiation. Thus, the radiation survival curves obtained using the colony formation assay showed that idasanutlin pretreatment sensitized human ATRT cells to ionizing radiation.

To investigate whether idasanutlin and radiation treatment in combination increased DNA damage, the BT16 cell line was cultured and treated with DMSO or idasanutlin $\left(\mathrm{IC}_{50}\right.$, $30 \mathrm{nM}$ ) and concurrently treated with 4 Gy radiation. After $24 \mathrm{~h}$, the cells were evaluated for $\gamma \mathrm{H} 2 \mathrm{AX}$ expression using immunofluorescence, as a surrogate marker of DNA damage. Compared with that in the DMSO control, idasanutlin alone increased the $\gamma \mathrm{H} 2 \mathrm{AX}$ signal in the ATRT cell line $(\mathrm{P}<0.01$; Fig. 6C and D). Radiation treatment alone also resulted in the accumulation of $\gamma \mathrm{H} 2 \mathrm{AX}$, which was significantly enhanced by idasanutlin in the ATRT cell line $(\mathrm{P}<0.005$; Fig. $6 \mathrm{C}$ and $\mathrm{D})$. The data were further confirmed by evaluating $\gamma \mathrm{H} 2 \mathrm{AX}$ using 
A
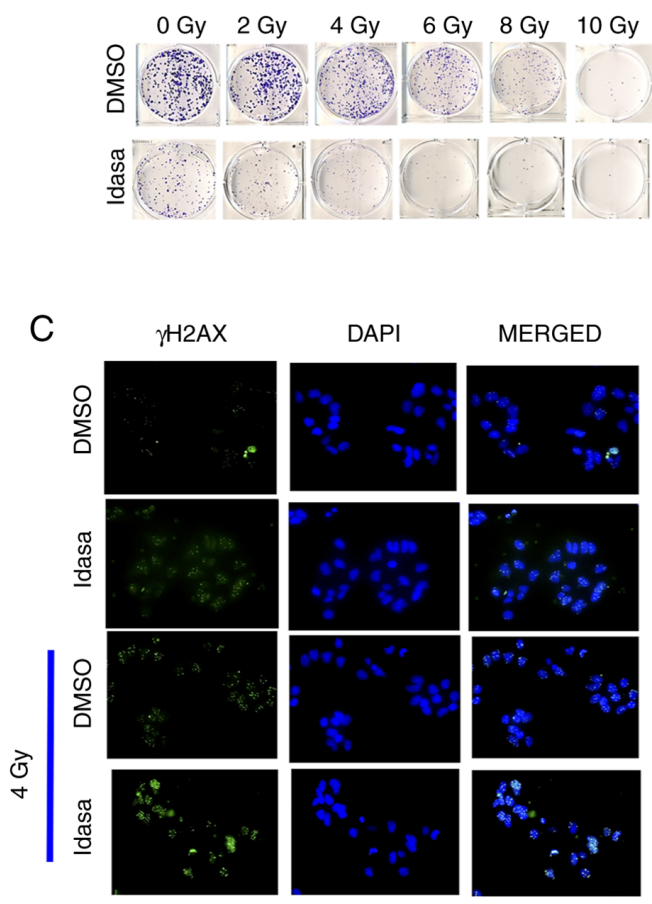

MERGED

E

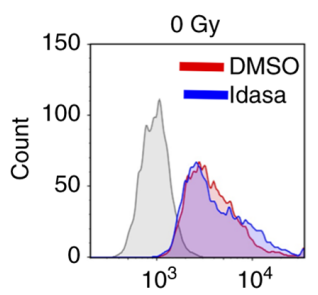

Intensity of Alexa Fluor 488- $\gamma \mathrm{H} 2 \mathrm{AX}$ (pS139)

$\mathrm{H}$

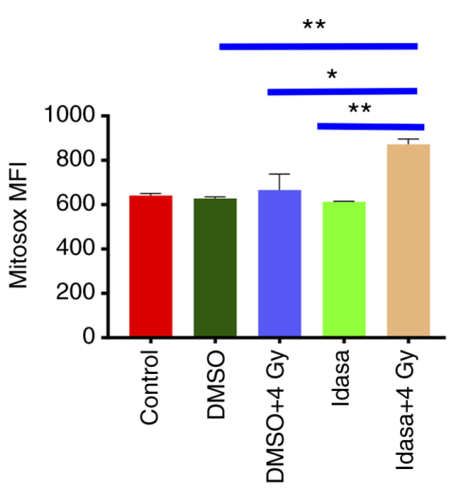

B

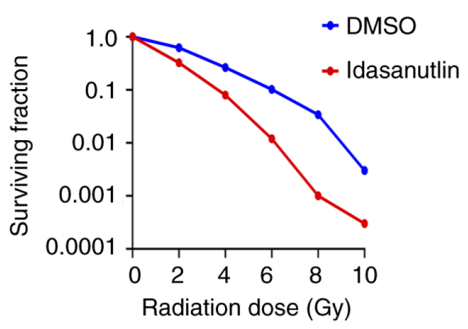

D

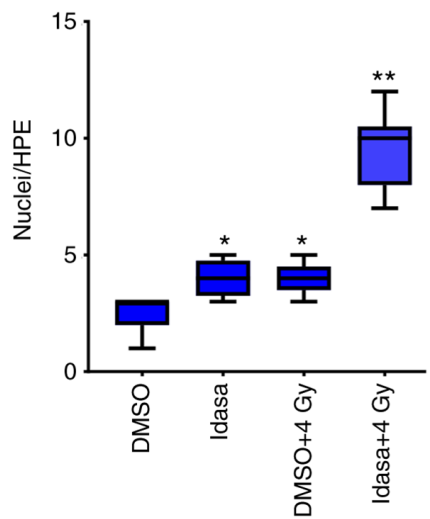

$\mathrm{F}$
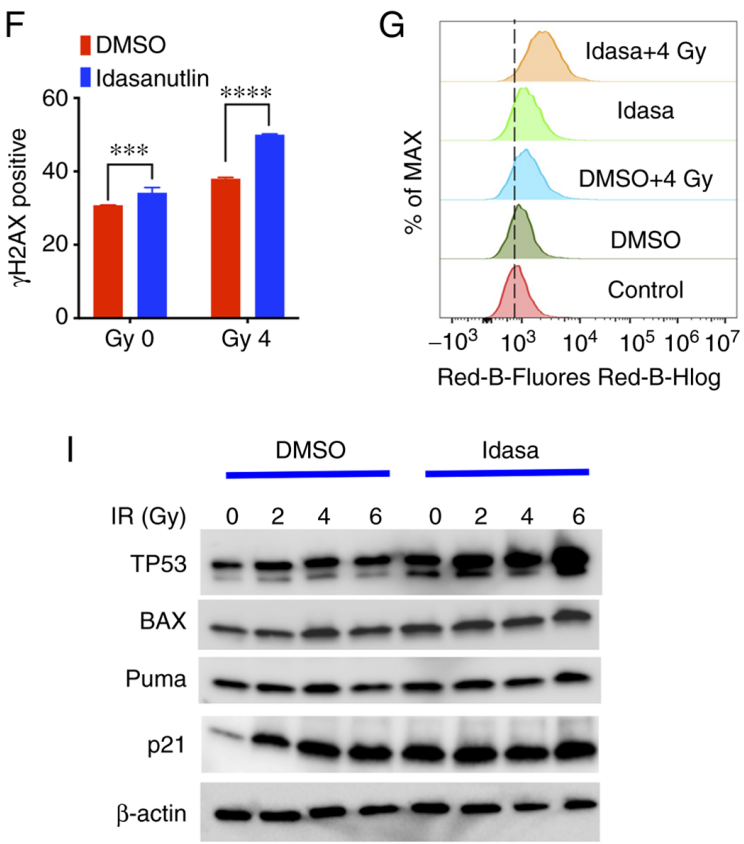

Figure 6. Idasanutlin sensitizes the BT16 ATRT cell line to radiation treatment. (A) The BT16 cell line was treated with different doses of radiation following $6 \mathrm{~h}$ pretreatment with $18 \mathrm{nM}$ idasanutlin, then 10 days later a colony formation assay was performed. (B) The surviving fraction was calculated 10 days later according to the number of colonies. Sensitivity enhancement ratio was calculated at 10 and $50 \%$ of the surviving fraction. Idasanutlin increased radiosensitization of BT16 ATRT cells. (C) Representative immunofluorescence images of $\gamma \mathrm{H} 2 \mathrm{AX}$ accumulation in the BT16 ATRT cells, following treatment with idasanutlin ( $\mathrm{IC5}_{0}, 30 \mathrm{nM} ; 72 \mathrm{~h}$ ), then radiated with 4 Gy radiation. The cells were stained with $\gamma \mathrm{H} 2 \mathrm{AX}$ and DAPI $24 \mathrm{~h}$ after radiation. (D) Quantification of immunofluorescence. ${ }^{*} \mathrm{P}<0.05 ;{ }^{* *} \mathrm{P}<0.001$ vs. DMSO. (E) Flow cytometry was used to analyze $\gamma \mathrm{H} 2 \mathrm{AX}$ accumulation in cells treated with idasanutlin (IC ${ }_{50}$, $30 \mathrm{nM} ; 72 \mathrm{~h}$ ), then radiation ( $4 \mathrm{~Gy} ; 24 \mathrm{~h}$ ), then the results were (F) quantified. $\left.{ }^{* * *} \mathrm{P}<0.001,{ }^{* * * *} \mathrm{P}<0.0001\right)$. (G) Flow cytometry was used to analyze reactive oxygen species in the BT16 ATRT cell line treated with idasanutlin ( $\mathrm{IC}_{50}, 30 \mathrm{nM} ; 72 \mathrm{~h}$ ), then radiated (4 Gy; $\left.24 \mathrm{~h}\right)$. (H) Quantification of ROS accumulation. ${ }^{*} \mathrm{P}<0.05$. ${ }^{* *} \mathrm{P}<0.01$. (I) Western blot analysis was used to analyze the expression level of different pro-apoptotic proteins in the BT16 ATRT cells following treatment with idasanutlin $\left(\mathrm{IC}_{50}, 30 \mathrm{nM} ; 72 \mathrm{~h}\right)$ in combination with 2, 4 and 6 Gy radiation. ATRT, atypical teratoid rhabdoid tumor; ROS, reactive oxygen species

flow cytometry (Fig. 6E and F). Idasanutlin significantly enhanced radiation-mediated DNA damage $(\mathrm{P}<0.0001$ Fig. 6F). Subsequently, it was investigated whether the DNA damage was associated with increased ROS, in response to radiation. ROS was analyzed using flow cytometry and the MitoSox assay (Fig. 6G and H). Treatment with idasanutlin 
and radiation combined significantly enhanced ROS formation, further indicating that MDM2 inhibition sensitizes ATRT cells to radiation (Fig. 6G and $\mathrm{H}$ ). Consistent with this data, treatment of the BT16 cells with idasanutlin $\left(\mathrm{IC}_{50}, 30 \mathrm{nM}\right)$ enhanced TP53 protein induction by radiation, concurrent with induction of the pro-apoptotic BAX and PUMA proteins, which are targets of TP53 activity (Figs. 6I, and S6 and S7). Together these data establish a strategy to treat ATRT with idasanutlin and radiation.

\section{Discussion}

Characterized by an average 5-year survival rate of 30-40\%, ATRT is a highly aggressive brain tumor, that develops in childhood $(2,36,37)$. Intensive chemotherapy, radiation and autologous stem cell transplant results in therapy-related toxicity with poor results $(3,4)$. Epigenetic dysregulation of the genome, caused by the loss of the SMARCB1 gene, is the key molecular feature in ATRT $(5,6)$. SMARCB1 affects lineage commitment and differentiation by controlling gene expression (7). ATRT demonstrate a quiescent genome, but gene expression analysis shows they are heterogeneous (8-11). A previous study demonstrated that the SMARCB1 deletion leads to assembly of aberrant SWI/SNF complexes, which results in differential occupancy of super-enhancers (38). Furthermore, SMARCB1 is required to activate bivalent promoter (39). Unfortunately, therapeutic strategies to target the deletion of SMARCB1 have remained elusive. Thus, there is an urgent requirement to improve the understanding of how SMARCB1 regulates tumorigenesis leading to novel, less toxic and more effective therapies for children with ATRT.

Using unbiased RNA interference screening, TP53 was identified as a potential therapeutic target in ATRT. TP53, as a critical component in SMARCB1 deficient tumors, is an emerging concept. In SMARCB1 deleted extra-cranial MRT, TP53 acts as a regulator of proteostasis and tumor progression (23). Carugo et al (23) demonstrated that the SMARCB1 deletion drives endoplasmi reticulum stress in murine livers and that attenuation of the autophagy response could suppress MRT formation. Importantly, they identified TP53 a key regulator of the autophagy response driven by SMARCB1 deficiency (23). A previous study showed that TP53 activation by MDM2/MDM4 inhibition was effective in SMARCB1 deleted malignant rhabdoid tumor cells and decreased the growth of flank xenografts in vivo (22). The results from that study combined with the results from the present study identifies a novel therapeutic strategy for both intra cranial and extra cranial SMARCB1 deleted rhabdoid tumors.

Howard et al (22) identified TP53 as a potential target in MRT using data from genome-wide CRISPR screens. In addition, SMARCB1 sufficiency regulates the response to TP53 driven apoptosis and that sensitivity to MDM2 inhibition is associated with expression in MRT cell lines (22).

ATRT subgroups (SHH, TYR and MYC), classified by DNA methylation and gene expression patterns, demonstrate differences in clinical presentation (2). TYR tumors are associated with a younger median age and infratentorial location, while SHH occur more frequently in the cerebral cortex (2). SMARCB1 alterations also vary between groups. SHH and TYR tumors are associated with point mutations and focal deletions, while MYC tumors exhibit broad deletion $(2,12)$. However, it is not yet clear if these subgroups affect the response to therapy. In the present study it was found that idasanutlin inhibits ATRT cell growth in all ATRT subgroups, but with different $\mathrm{IC}_{50}$ concentrations. The highest $\mathrm{IC}_{50}$ was found with the lowest MDM2 expression among the ATRT subgroups. Furthermore, idasanutlin inhibited in vivo growth in orthotopic intracranial xenografts by activating the TP53 pathway. Furthermore, chemical inhibition of MDM2 co-operates with radiation to enhance DNA damage and cell death in vitro. Additional in vivo studies are required to establish optimal dosing schedules, combining idasanutlin with radiation. The results from the present study establish TP53 as a druggable pathway in ATRT. In glioblastoma, RG7388 suppressed tumor growth in vitro and in vivo, but resistance occurred in the MAP kinase pathway (40). Similarly P53/MDM2 inhibition synergizes with EGFR inhibition in glioblastoma (41). While glioblastoma is genetically diverse and distinct from SMARCB1 deficient ATRT, these 2 studies $(40,41)$ demonstrate the generalizability of p53/MDM2 inhibition in brain tumors.

Idasanutlin has been examined in a range of adult clinical trials, targeting hematopoietic and solid tumors (NCT02828930, NCT01773408 and NCT01901172). The major dose limiting toxicities identified in these studies included myelosuppression (42). Currently, there are several ongoing studies in leukemia and solid tumors (43). Of note, a study investigating idasanutlin in brain tumors is currently open for glioblastoma (NCT03158389). However, no studies investigating idasanutlin are open in pediatric brain tumors. Several pediatric clinical trials consortia are planning novel ATRT trials by adding agents to current chemotherapy backbones. DM2 inhibition is one of these approaches being considered and several trials in ATRT are planned (Dr Susan Chi, Pediatrics, Boston Children's Hospital; personal communication).

The results from the present study, combined with that from Howard et al (22) established a novel therapy for SMARCB1 deficient rhabdoid tumors, irrespective of tumor location or genomic subgroup and provide the preclinical justification for trials in pediatric tumors. Notably, the Pediatric Preclinical Testing Program found RG7112 (an earlier generation of a MDM2 inhibitor) was effective against non-cranial malignant rhabdoid tumors, further confirming the role of MDM2/TP53 in SMARCB1 deficient tumors (43). These studies warrant further investigation and clinical development for ATRT and other pediatric brain tumors.

In conclusion, the present study shows that MDM2 is a therapeutic target in ATRT. In vivo pharmacological inhibition of MDM2 using idasanutlin significantly decreases growth of intracranial orthotopic ATRT tumors, leads to necrosis/apoptosis in ATRT tumors and prolongs survival time in idasanutlin treated mice. More importantly, it was found that idasanutlin sensitizes ATRT cells to ionizing radiation by increasing DNA damage and ROS accumulation in vitro. It was demonstrated that the TP53/MDM2 axis is a crucial regulator of ATRT cell growth, as well as a potential target in this childhood brain tumor. 


\section{Acknowledgements}

The authors appreciate the contribution to this research made by E. Erin Smith, Allison Quador, and Jessica Arnold, from the University of Colorado Pathology Shared Resource, Research Histology Division (Colorado, USA). The authors would also like to thank Natalie Serkova and Jenna Steiner from the University of Colorado Animal Imaging Shared Resource (Colorado, USA) for acquiring all mouse MRI scans and data analysis. This study was presented in part at the 6th Biennial Pediatric Neuro-Oncology Research Conference, June 0-12, 2021 (Neuro-Oncology, 2021, Vol 3 (Supplement_1), pp. i2-i3).

\section{Funding}

This study was supported by the Morgan Adams Foundation Pediatric Research Program and DOD grant (grant. no. CA170677). The University of Colorado Pathology Shared Resource, Research Histology Division (Colorado, USA) was supported in part by the Cancer Center Support Grant (P30CA046934). All imaging protocols were developed at the Colorado Animal Imaging Shared Resource supported by the NIH Shared Instrumentation Grant Program (S10 OD023485, S10 OD027023), and the University of Colorado Cancer Center grant (P30 CA046934).

\section{Availability of data and materials}

Publicly available datasets were analyzed in the present study. The data can be found on the Gene Expression Omnibus database (GSE70678). All the data used and/or generated in the current study are available from the corresponding author upon reasonable request.

\section{Authors' contributions}

RV and IA designed the study and wrote the manuscript. IA conducted the in vitro and in vivo experiments, performed data analysis, and prepared the figures. AP developed and performed the in vivo studies. ED and DW performed data analysis. AD quantified and analyzed immunohistochemistry images and contributed to the design of the study, and the draft version of the manuscript. NS analyzed the MRI data. KM developed and performed radiation studies. IB developed the colony formation assay and quantification methods. SL performed the MitoSOX assay. SM provided input on ATRT cell biology, contributed to the design of the study, assisted with writing the manuscript, made substantial contributions to conception of the manuscript. SV, NKF and RV conceived the project and edited the manuscript. IA and RV confirm the authenticity of all the raw data. All authors were involved in the writing, reviewing and editing the manuscript. All authors have read and agreed to the published version of the manuscript.

\section{Ethics approval and consent to participate}

The protocol was approved by the Ethics Committee of University of Colorado and Children's Hospital Colorado (approval number, COMIRB 95-500) All patients or parents/guradians provided written informed consent for molecular studies of their tumor.

\section{Patient consent for publication}

Not applicable.

\section{Competing interests}

The authors declare that they have no competing interests.

\section{References}

1. Frühwald MC, Biegel JA, Bourdeaut F, Roberts CW and Chi SN: Atypical teratoid/rhabdoid tumors-current concepts, advances in biology, and potential future therapies. Neuro Oncol 18: 764-778, 2016.

2. Hoffman LM, Richardson EA, Ho B, Margol A, Reddy A, Lafay-Cousin L, Chi S, Slave I, Judkins A, Hasselblatt M, et al: Advancing biology-based therapeutic approaches for atypical teratoid rhabdoid tumors. Neuro Oncol 22: 944-954, 2020.

3. Chi SN, Zimmerman MA, Yao X, Cohen KJ, Burger P, Biegel JA, Rorke-Adams LB, Fisher MJ, Janss A, Mazewski C, et al: Intensive multimodality treatment for children with newly diagnosed CNS atypical teratoid rhabdoid tumor. J Clin Oncol 27: 385-389, 2009.

4. Lafay-Cousin L, Fay-McClymont T, Johnston D, Fryer C, Scheinemann K, Fleming A, Hukin J, Janzen L, Guger S, Strother D, et al: Neurocognitive evaluation of long term survivors of atypical teratoid rhabdoid tumors (ATRT): The Canadian registry experience. Pediatr Blood Cancer 62: 1265-1269, 2015.

5. Biegel JA, Tan L, Zhang F, Wainwright L, Russo P and Rorke LB: Alterations of the hSNF5/INI1 gene in central nervous system atypical teratoid/rhabdoid tumors and renal and extrarenal rhabdoid tumors. Clin Cancer Res 8: 3461-3467, 2002.

6. Biegel JA, Zhou JY, Rorke LB, Stenstrom C, Wainwright LM and Fogelgren B: Germ-line and acquired mutations of INI1 in atypical teratoid and rhabdoid tumors. Cancer Res 59: 74-79, 1999.

7. Kadoch $\mathrm{C}$ and Crabtree GR: Mammalian SWI/SNF chromatin remodeling complexes and cancer: Mechanistic insights gained from human genomics. Sci Adv 1: e1500447, 2015.

8. Torchia J, Golbourn B, Feng S, Ho KC, Sin-Chan P, Vasiljevic A, Norman JD, Guilhamon P, Garzia L, Agamez NR, et al: Integrated (epi)-genomic analyses identify subgroup-specific therapeutic targets in CNS rhabdoid tumors. Cancer Cell 30: 891-908, 2016

9. Johann PD, Erkek S, Zapatka M, Kerl K, Buchhalter I, Hovestadt V, Jones DTW, Sturm D, Hermann C, Segura Wang M, et al: Atypical teratoid/rhabdoid tumors are comprised of three epigenetic subgroups with distinct enhancer landscapes. Cancer Cell 29: 379-393, 2016.

10. Lee RS, Stewart C, Carter SL, Ambrogio L, Cibulskis K, Sougnez C, Lawrence MS, Auclair D, Mora J, Golub TR, et al: A remarkably simple genome underlies highly malignant pediatric rhabdoid cancers. J Clin Invest 122: 2983-2988, 2012.

11. Birks DK, Donson AM, Patel PR, Dunham C, Muscat A, Algar EM, Ashley DM, Kleinschmidt-Demasters BK, Vibhakar R, Handler MH and Foreman NK: High expression of BMP pathway genes distinguishes a subset of atypical teratoid/rhabdoid tumors associated with shorter survival. Neuro Oncol 13: 1296-1307, 2011.

12. Ho B, Johann PD, Grabovska Y, De Dieu Andrianteranagna MJ, Yao F, Frühwald M, Hasselblatt M, Bourdeaut F, Williamson D, Huang A and Kool M: Molecular subgrouping of atypical teratoid/rhabdoid tumors-a reinvestigation and current consensus. Neuro Oncol 22: 613-624, 2020.

13. Theruvath J, Sotillo E, Mount CW, Graef CM, Delaidelli A, Heitzeneder S, Labanieh L, Dhingra S, Leruste A, Majzner RG, et al: Locoregionally administered B7-H3-targeted CAR T cells for treatment of atypical teratoid/rhabdoid tumors. Nat Med 26: 712-719, 2020 
14. Birks DK, Donson AM, Patel PR, Sufit A, Algar EM, Dunham C, Kleinschmidt-DeMasters BK, Handler MH, Vibhakar R and Foreman NK: Pediatric rhabdoid tumors of kidney and brain show many differences in gene expression but share dysregulation of cell cycle and epigenetic effector genes. Pediatr Blood Cancer 60: 1095-1102, 2013

15. Wade M, Li YC and Wahl GM: MDM2, MDMX and p53 in oncogenesis and cancer therapy. Nat Rev Cancer 13: 83-96, 2013.

16. Tovar C, Graves B, Packman K, Filipovic Z, Higgins B, Xia M, Tardell C, Garrido R, Lee E, Kolinsky K, et al: MDM2 small-molecule antagonist RG7112 activates p53 signaling and regresses human tumors in preclinical cancer models. Cancer Res 73: 2587-2597, 2013.

17. Shangary $S$ and Wang S: Small-molecule inhibitors of the MDM2-p53 protein-protein interaction to reactivate p53 function: A novel approach for cancer therapy. Annu Rev Pharmacol Toxicol 49: 223-241, 2009.

18. Vassilev LT, Vu BT, Graves B, Carvajal D, Podlaski F, Filipovic Z, Kong N, Kammlott U, Lukacs C, Klein C, et al: In vivo activation of the p53 pathway by small-molecule antagonists of MDM2. Science 303: 844-848, 2004.

19. Ding Q, Zhang Z, Liu JJ, Jiang N, Zhang J, Ross TM, Chu XJ, Bartkovitz D, Podlaski F, Janson C, et al: Discovery of RG7388, a potent and selective p53-MDM2 inhibitor in clinical development. J Med Chem 56: 5979-5983, 2013.

20. Chen L, Rousseau RF, Middleton SA, Nichols GL, Newell DR, Lunec $J$ and Tweddle DA: Pre-clinical evaluation of the MDM2-p53 antagonist RG7388 alone and in combination with chemotherapy in neuroblastoma. Oncotarget 6: 10207-10221, 2015.

21. Lakoma A, Barbieri E, Agarwal S, Jackson J, Chen Z, Kim Y, McVay M, Shohet JM and Kim ES: The MDM2 small-molecule inhibitor RG7388 leads to potent tumor inhibition in p53 wild-type neuroblastoma. Cell Death Discov 1: 15026, 2015.

22. Howard TP, Arnoff TE, Song MR, Giacomelli AO, Wang X, Hong AL, Dharia NV, Wang S, Vazquez F, Pham MT, et al: MDM2 and MDM4 Are therapeutic vulnerabilities in malignant rhabdoid tumors. Cancer Res 79: 2404-2414, 2019.

23. Carugo A, Minelli R, Sapio L, Soeung M, Carbone F, Robinson FS, Tepper J, Chen Z, Lovisa S, Svelto M, et al: p53 is a master regulator of proteostasis in SMARCB1-deficient malignant rhabdoid tumors. Cancer Cell 35: 204-220.e9, 2019.

24. Alimova I, Pierce AM, Harris P, Donson A, Birks DK, Prince E, Balakrishnan I, Foreman NK, Kool M, Hoffman L, et al Targeting Polo-like kinase 1 in SMARCB1 deleted atypical teratoid rhabdoid tumor. Oncotarget 8: 97290-97303, 2017.

25. Alimova I, Pierce A, Danis E, Donson A, Birks DK, Griesinger A, Foreman NK, Santi M, Soucek L, Venkataraman S and Vibhakar R: Inhibition of MYC attenuates tumor cell self-renewal and promotes senescence in SMARCB1-deficient group 2 atypical teratoid rhabdoid tumors to suppress tumor growth in vivo. Int J Cancer 144: 1983-1995, 2019.

26. Tang Y, Gholamin S, Schubert S, Willardson MI, Lee A, Bandopadhayay P, Bergthold G, Masoud S, Nguyen B, Vue N, et al: Epigenetic targeting of Hedgehog pathway transcriptional output through BET bromodomain inhibition. Nat Med 20: 732-740, 2014.

27. Balakrishnan I, Danis E, Pierce A, Madhavan K, Wang D, Dahl N, Sanford B, Birks DK, Davidson N, Metselaar DS, et al: Senescence induced by BMI1 inhibition is a therapeutic vulnerability in H3K27M-mutant DIPG. Cell Rep 33: 108286, 2020.

28. Gautier L, Cope L, Bolstad BM and Irizarry RA: affy-analysis of Affymetrix GeneChip data at the probe level. Bioinformatics 20 : 307-315, 2004
29. Dai M, Wang P, Boyd AD, Kostov G, Athey B, Jones EG, Bunney WE, Myers RM, Speed TP, Akil H, et al: Evolving gene/transcript definitions significantly alter the interpretation of GeneChip data. Nucleic Acids Res 33: e175, 2005.

30. Kauffmann A, Gentleman R and Huber W: arrayQualityMetrics-a bioconductor package for quality assessment of microarray data. Bioinformatics 25: 415-416, 2009.

31. Irizarry RA, Hobbs B, Collin F, Beazer-Barclay YD, Antonellis KJ, Scherf U and Speed TP: Exploration, normalization, and summaries of high density oligonucleotide array probe level data. Biostatistics 4: 249-264, 2003.

32. Wang D, Pierce A, Veo B, Fosmire S, Danis E, Donson A, Venkataraman $S$ and Vibhakar R: A regulatory loop of FBXW7-MYC-PLK1 controls tumorigenesis of MYC-driven medulloblastoma. Cancers (Basel) 13: 387, 2021.

33. Alimova I, Ng J, Harris P, Birks D, Donson A, Taylor MD, Foreman NK, Venkataraman S and Vibhakar R: MPS1 kinase as a potential therapeutic target in medulloblastoma. Oncol Rep 36: 2633-2640, 2016

34. Kauffman ME, Kauffman MK, Traore K, Zhu H, Trush MA, Jia Z and Li YR: MitoSOX-based flow cytometry for detecting mitochondrial ROS. React Oxyg Species (Apex) 2: 361-370, 2016.

35. Barretina J, Caponigro G, Stransky N, Venkatesan K, Margolin AA, Kim S, Wilson CJ, Lehár J, Kryukov GV, Sonkin D, et al: The cancer cell line encyclopedia enables predictive modelling of anticancer drug sensitivity. Nature 483: 603-607, 2012

36. Buscariollo DL, Park HS, Roberts KB and Yu JB: Survival outcomes in atypical teratoid rhabdoid tumor for patients undergoing radiotherapy in a surveillance, epidemiology, and end results analysis. Cancer 118: 4212-4219, 2012.

37. Rorke LB, Packer RJ and Biegel JA: Central nervous system atypical teratoid/rhabdoid tumors of infancy and childhood: Definition of an entity. J Neurosurg 85: 56-65, 1996.

38. Wang $X$, Lee RS, Alver BH, Haswell JR, Wang S, Mieczkowski J, Drier Y, Gillespie SM, Archer TC, Wu JN, et al: SMARCB1-mediated SWI/SNF complex function is essential for enhancer regulation. Nat Genet 49: 289-295, 2017.

39. Nakayama RT, Pulice JL, Valencia AM, McBride MJ, McKenzie ZM, Gillespie MA, Ku WL, Teng M, Cui K, Williams RT, et al: SMARCB1 is required for widespread BAF complex-mediated activation of enhancers and bivalent promoters. Nat Genet 49: 1613-1623, 2017.

40. Berberich A, Kessler T, Thomé CM, Pusch S, Hielscher T, Sahm F, Oezen I, Schmitt LM, Ciprut S, Hucke N, et al: Targeting resistance against the MDM2 inhibitor RG7388 in glioblastoma cells by the MEK inhibitor trametinib. Clin Cancer Res 25: 253-265, 2019.

41. Mai WX, Gosa L, Daniels VW, Ta L, Tsang JE, Higgins B, Gilmore WB, Bayley NA, Harati MD, Lee JT, et al: Cytoplasmic p53 couples oncogene-driven glucose metabolism to apoptosis and is a therapeutic target in glioblastoma. Nat Med 23: 1342-1351, 2017

42. Konopleva M, Martinelli G, Daver N, Papayannidis C, Wei A, Higgins B, Ott M, Mascarenhas J and Andreeff M: MDM2 inhibition: An important step forward in cancer therapy. Leukemia 34: 2858-2874, 2020

43. Carol H, Reynolds CP, Kang MH, Keir ST, Maris JM, Gorlick R Kolb EA, Billups CA, Geier B, Kurmasheva RT, et al: Initial testing of the MDM2 inhibitor RG7112 by the pediatric preclinical testing program. Pediatr Blood Cancer 60: 633-641, 2013.

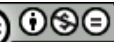

This work is licensed under a Creative Commons Attribution-NonCommercial-NoDerivatives 4.0 International (CC BY-NC-ND 4.0) License. 